\title{
INL REPORT
}

INL/EXT-17-44085

Unlimited Release

Printed December 2017

\section{Validation of Pronghorn with the SANA Experiments}

Ling Zou

April J. Novak

Richard C. Martineau

Hans D. Gougar

Prepared by

Idaho National Laboratory

Idaho Falls, Idaho 83415

The Idaho National Laboratory is a multiprogram laboratory operated by

Battelle Energy Alliance for the United States Department of Energy

under DOE Idaho Operations Office. Contract DE-AC07-05ID14517.

Approved for public release; further dissemination unlimited.

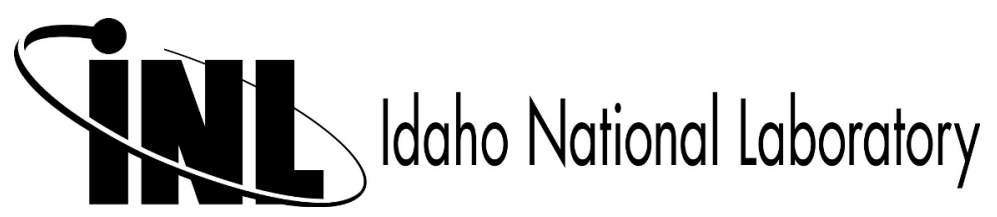


Issued by the Idaho National Laboratory, operated for the United States Department of Energy by Battelle Energy Alliance.

NOTICE: This report was prepared as an account of work sponsored by an agency of the United States Government. Neither the United States Government, nor any agency thereof, nor any of their employees, nor any of their contractors, subcontractors, or their employees, make any warranty, express or implied, or assume any legal liability or responsibility for the accuracy, completeness, or usefulness of any information, apparatus, product, or process disclosed, or represent that its use would not infringe privately owned rights. Reference herein to any specific commercial product, process, or service by trade name, trademark, manufacturer, or otherwise, does not necessarily constitute or imply its endorsement, recommendation, or favoring by the United States Government, any agency thereof, or any of their contractors or subcontractors. The views and opinions expressed herein do not necessarily state or reflect those of the United States Government, any agency thereof, or any of their contractors.

Printed in the United States of America. This report has been reproduced directly from the best available copy.

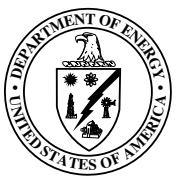


INL/EXT-17-44085

Unlimited Release

Printed December 2017

\title{
Validation of Pronghorn with the SANA Experiments
}

\author{
Ling Zou \\ Idaho National Laboratory \\ P.O. Box 1625 \\ Idaho Falls, ID 83415-3870 \\ April J. Novak \\ University of California, Berkeley \\ Richard C. Martineau \\ Idaho National Laboratory \\ Hans D. Gougar \\ Idaho National Laboratory
}




\section{Contents}

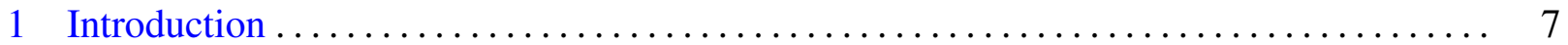

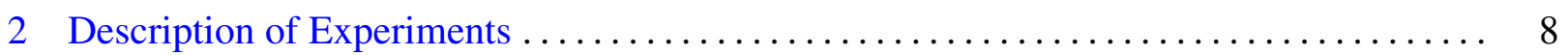

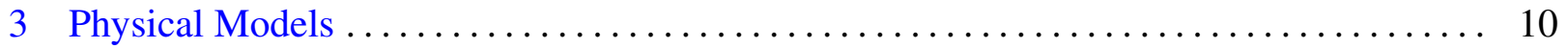

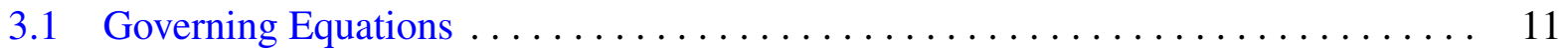

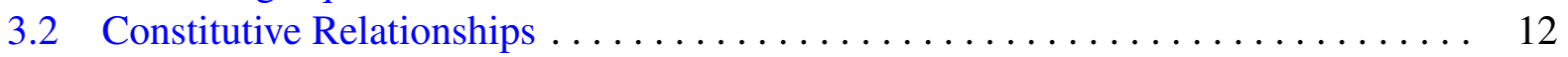

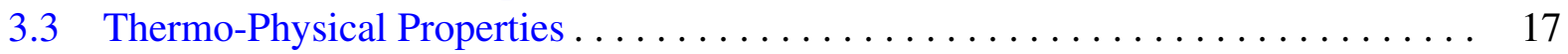

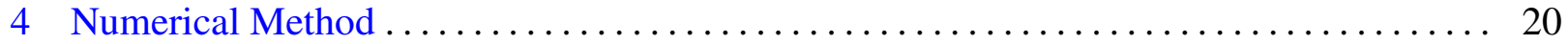

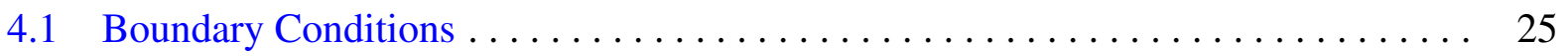

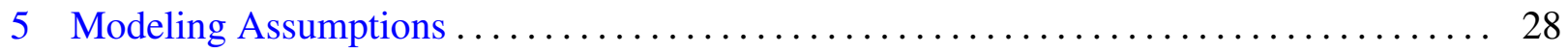

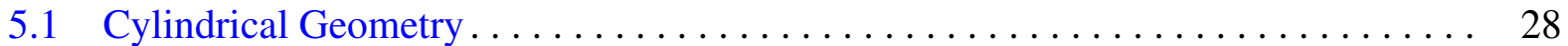

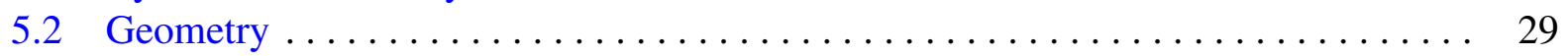

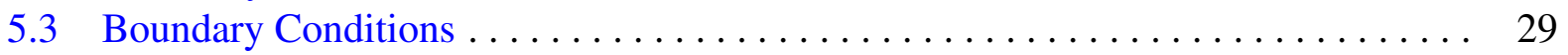

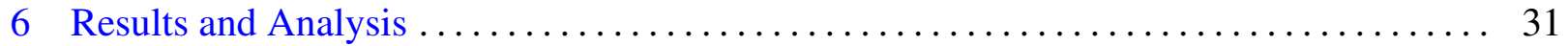

6.1 Numerical Verification $\ldots \ldots \ldots \ldots \ldots \ldots \ldots \ldots \ldots \ldots \ldots \ldots \ldots \ldots \ldots \ldots$

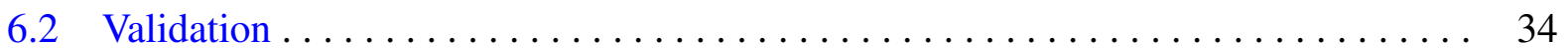

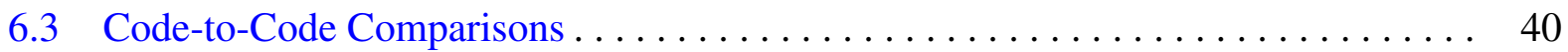

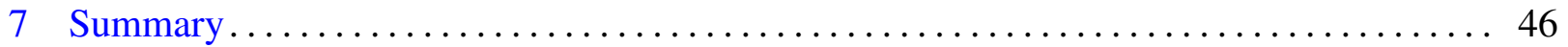

\section{Appendix}

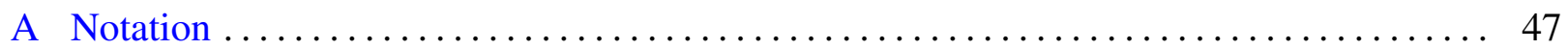

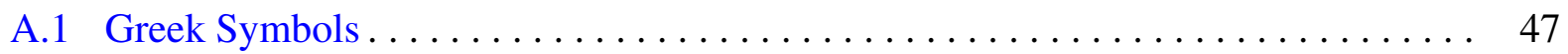

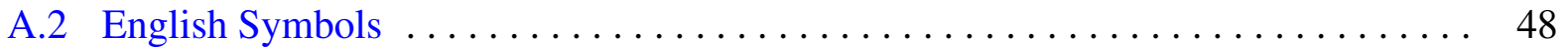

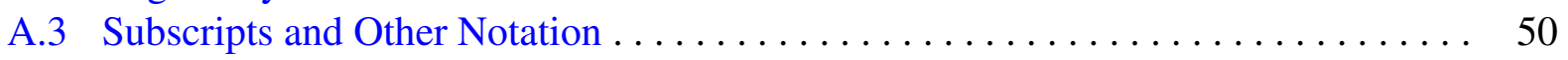

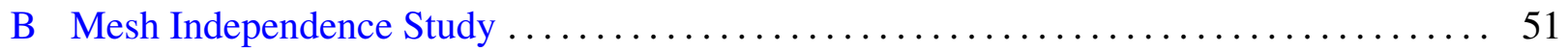

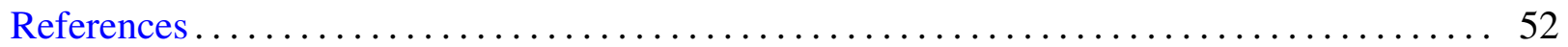




\section{Figures}

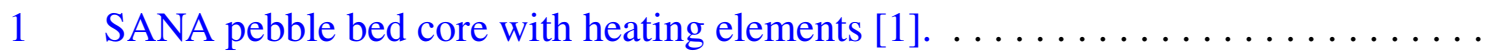

2 Validation of Zehner-Bauer-Schlünder correlation (pronghorn) predicted effective thermal conductivity values compared to experimental measurements and THER-

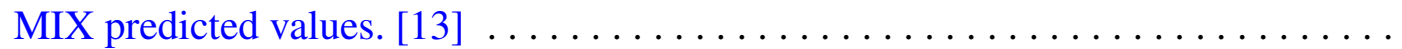

3 Error as a function of mesh spacing $\Delta x$ for $\varepsilon=0.35, W=2.0$, and (a) $P=1000 x^{2}+$ $50 e^{y}$ and $T_{f}=1000$, (b) $P=1000 x^{3}+50 e^{y}$ and $T_{f}=50 e^{x}+1000 y^{3}+2000$, and (c) $P=100 e^{x}+200 y+500$ and $T_{f}=1000+50 e^{y}$ for the pressure Poisson kernel [4]. 32

4 Error as a function of mesh spacing $\Delta x$ for (a) $T_{f}=\sin (2 \pi x) \sin (2 \pi y)$ and $\kappa=$ $100 x=200 y+5000$, (b) $T_{f}=e^{x} e^{y}$ and $\kappa=200 x^{3}+300 y^{4}$, and (c) $T_{f}=x^{3}+e^{y}$ and $\kappa=\sin (x)+\cos (y)$ for the solid energy diffusion kernel in 2-D $r$ - $z$ geometry [4]. 33

5 Convergence rates for different time integration schemes for $T_{f}=(x+y+15) e^{-1}$, $\rho_{f}=100 x+200 y+1, \varepsilon=0.35 x+0.25 y+0.1$, and $C_{p}=e^{x}+e^{y}+100$ for the $\left(\varepsilon \rho_{f} C_{p, f} \frac{\partial T_{f}}{\partial t}, \psi\right)$ kernel [4]. This is the time derivative kernel in the fluid energy equation.

6 Pronghorn solid temperature computed for helium with $10 \mathrm{~kW}$ nominal heater rod power (case A). . . . . . . . . . . . . . . . . . . . . . . .

7 Pronghorn solid temperature computed for helium with $20 \mathrm{~kW}$ nominal heater rod power (case B). . . . . . . . . . . . . . . . . . . . . . . . .

8 Pronghorn solid temperature computed for helium with $30 \mathrm{~kW}$ nominal heater rod

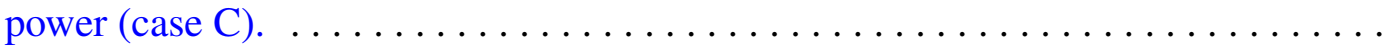

9 Pronghorn solid temperature computed for nitrogen with $10 \mathrm{~kW}$ nominal heater

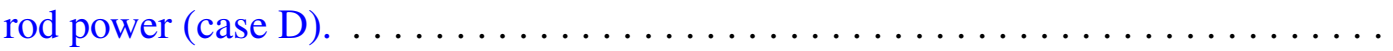

10 Pronghorn solid temperature computed for nitrogen with $20 \mathrm{~kW}$ nominal heater

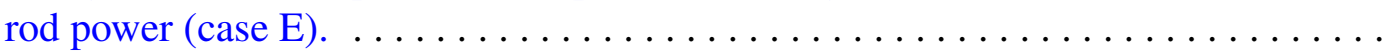

11 Pronghorn solid temperature computed for nitrogen with $25 \mathrm{~kW}$ nominal heater

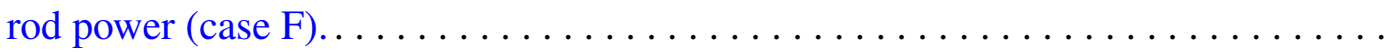

12 Meshes used for SANA experiment simulations for (a) the helium cases, and (b)

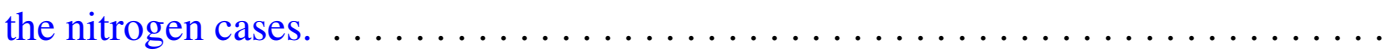

13 Pronghorn results for the velocity field of case A $(10 \mathrm{~kW}$ nominal heating power and helium coolant). The plot is colored with velocity magnitude in $(\mathrm{m} / \mathrm{s}) \ldots \ldots$.

14 Legacy Pronghorn temperature results for case A $[21] \ldots \ldots \ldots \ldots \ldots \ldots$.

15 MGT-3D and ANSYS CFX (CFD) temperature results for case C [19]. The uncertainty band was estimated by varying the Boundary Condition (BC)s within the bounds recommended in the SANA documents. . . . . . . . . . . . . . 42

16 THERMIX temperature results for case A [13]. . . . . . . . . . . . . 43

17 TRIO-EF temperature results for case A $[13] \ldots \ldots \ldots \ldots \ldots \ldots \ldots \ldots \ldots \ldots$

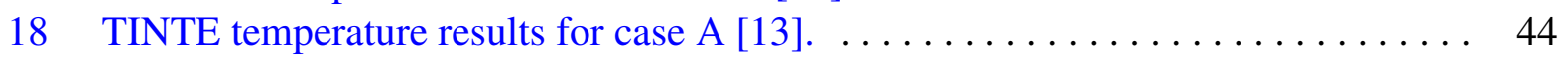

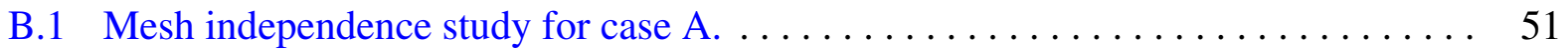




\section{Tables}

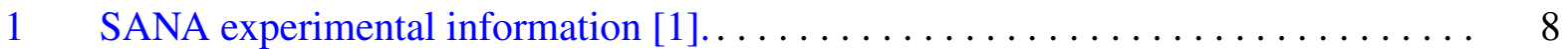

2 SANA experiments addressed in this report. . . . . . . . . . . . . 9

3 Boundary conditions used for the SANA experiment cases described in Table 2. . . 30 


\section{Introduction}

Pebble bed High Temperature Reactor (HTR)s are expected to display excellent heat removal characteristics in operational and accident scenarios due to graphite's capability for storing and transferring heat, the very high failure temperatures of particle fuel, and the low power densities involved. In order to validate the expected thermal stability and heat removal characteristics of pebble bed HTRs, a large experimental validation program was conducted in Germany from 1994 to 1996, i.e., the SANA experiment [1]. This experiment has been widely used as a benchmark case to support code developments intended for thermal-hydraulics analysis of pebble bed HTRs. This report describes the validation of Pronghorn with a subset of the experiments in the SANA experimental database.

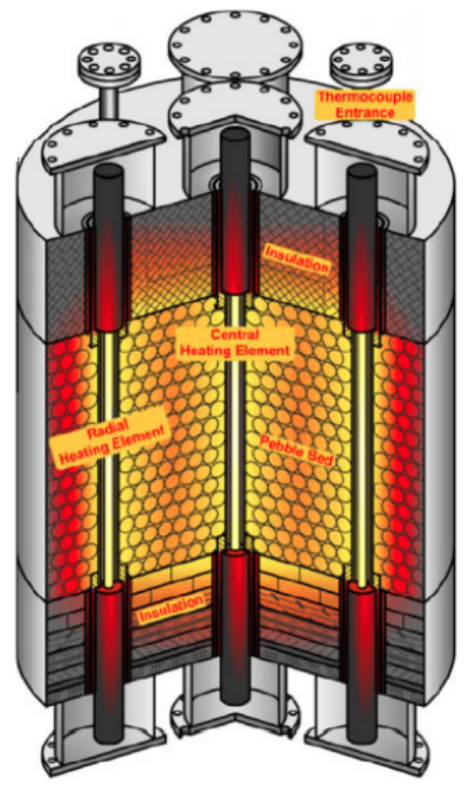

Figure 1. SANA pebble bed core with heating elements [1]. 


\section{Description of Experiments}

The experimental facility consists of a steel vessel containing a cylindrical bed of about 9500 spherical pebbles made of electric graphite, matrix graphite, or $\mathrm{Al}_{2} \mathrm{O}_{3}$. The bed was heated by one to four electric resistance heater elements and the temperature measured throughout the bed with a collection of thermocouples. Fig. 1 shows a schematic of the cylindrical bed with three resistance heaters shown.

The maximum installed power capacity is $50 \mathrm{~kW}$. The electrical resistance heater located in the center of the bed is $3.2 \mathrm{~cm}$ in diameter, and is protected by a tube of $14.1 \mathrm{~cm}$ outer diameter. The outer surface of the steel vessel is enclosed in a layer reducing the potential for accidental harm upon touch.

Either nitrogen, helium, or argon flow through the bed as coolant. A potential accident scenario for HTRs is an air ingress accident, whereupon graphite oxidation and corrosion can results in changes to core structural and fuel materials. To asses thermal-hydraulic performance under these conditions, nitrogen is used as a surrogate for air in the SANA experiments. Insulation is present at the top and bottom of the bed, and sometimes on the radial bed surface, in order to obtain desired heat fluxes. On the top of the bed, CERACHEM-Blanket material insulation of $40.0 \mathrm{~cm}$ is used, while on the bottom, six insulation layers are used of various materials to help support the weight of the bed, with a total thickness of $40.3 \mathrm{~cm}$. Correlations are provided with the benchmark specifications for the thermal conductivities of these various materials [1].

The maximum power density of $28 \mathrm{~kW} / \mathrm{m}^{3}$ corresponds to $0.93 \%$ of the full power of the AVR pebble bed reactor design, or the decay heat about 3 to 4 hours after shutdown of a typical Very High Temperature Reactor (VHTR). Table 1 provides important experimental information for the SANA experiments.

Table 1. SANA experimental information [1].

\begin{tabular}{ll}
\hline \hline Parameter & Value \\
\hline Bed height & $1.0 \mathrm{~m}$ \\
Bed inner radius & $0.0705 \mathrm{~m}$ \\
Bed outer radius & $0.75 \mathrm{~m}$ \\
Maximum installed power & $50 \mathrm{~kW}$ \\
Maximum power density & $28 \mathrm{~kW} / \mathrm{m}^{3}$ \\
\hline
\end{tabular}

Over 50 experiments were carried out for the three coolants and three types of pebbles. Steady state and transient temperatures, as well as values for effective solid thermal conductivity, were measured. Between one and four heaters were used; some tests use shortened heaters to simulate heat generation in only the upper or lower part of the bed. In several tests, the bed is only partway filled to simulate an upper plenum. This report focuses on the validation of Pronghorn using only a subset of the total number of experiments completed. The table below summarizes the SANA test cases that are discussed in this report. The letters in the leftmost column are used to refer to these 
particular cases throughout this report. All test cases are performed with the $6 \mathrm{~cm}$ electric graphite pebbles with a single, centrally-located heating element, in steady-state.

Table 2. SANA experiments addressed in this report.

\begin{tabular}{clccc}
\hline \hline Case & Coolant & Nominal Power $(\mathrm{kW})$ & Actual Power $(\mathrm{kW})$ & Ambient Temperature $\left({ }^{\circ} \mathrm{C}\right)$ \\
\hline A & helium & 10.00 & 8.91 & 23.0 \\
B & helium & 20.00 & 18.12 & 27.0 \\
C & helium & 35.10 & 27.42 & 26.0 \\
D & nitrogen & 10.03 & 8.91 & 26.3 \\
E & nitrogen & 20.00 & 18.12 & 25.6 \\
F & nitrogen & 24.97 & 22.74 & 29.5 \\
\hline
\end{tabular}




\section{Physical Models}

Numerical solutions to the flow equations for Reynolds numbers of interest for nuclear reactor applications are very computationally expensive due to the need to resolve thin boundary layers, capture fine-scale turbulent motions, and, depending on the numerical method, ensure stability. This computational cost can be prohibitive to accelerated design and analysis of engineering-scale phenomena such as reactor response to a loss of offsite power. Provided the effects of turbulence and viscosity can be approximately accounted for through appropriate empirical correlations, a simpler set of equations can be solved to predict reactor response at a coarser level. Porous media models, originally developed in the study of groundwater flows, approximate a solid-fluid medium as a two-phase mixture of solid and fluid, where the porosity $\varepsilon$ reflects the fraction of a representative volume that is fluid.

$$
\varepsilon=\frac{\text { fluid volume }}{\text { total volume }}
$$

The porosity in a cylindrical packed bed of spheres is a damped oscillatory function of the distance from the bounding wall [2]. Within a span of several pebbles, the porosity changes from unity at the wall to a typical bed average value in the range $0.35-0.45$. This large variation induces several important effects on the fluid flow. The pressure drop decreases linearly with porosity, and a $1 \%$ change in porosity produces about a $10 \%$ change in the local pressure drop [3]. The lower-porosity region near the bounding walls therefore leads to a flow-channeling effect, where velocities are higher near the core walls than in the center of the bed. The variation of pressure drop with porosity is captured in a porous medium friction factor.

In addition to porous media drag, the porosity influences the convective and conductive heat transfer in the bed. Lower porosities yield improved convective heat transfer due to the more tortuous fluid paths. The convective heat transfer coefficient decreases approximately linearly with porosity. While the correlation between porosity and convective heat transfer coefficient is not as strong as the correlation between porosity and pressure drop, a $1 \%$ change in the porosity produces about a 3\% change in the local convective heat transfer coefficient [3]. Finally, the solid conductive heat transfer is also a function of porosity. While conduction occurs within each solid pebble, conduction also occurs through pebble contact areas and by radiation across fluid gaps. Higher porosities therefore lead to improved radiation heat transfer, but reduced contact conduction.

The porous media versions of the Navier-Stokes equations are derived by averaging the equations over a representative volume consisting of a mixture of solid and fluid [4]. This averaging process produces several constitutive terms that are not normally present in the Navier-Stokes equations. A large number of experiments have been conducted to provide correlations for these constitutive terms, and are represented by the porous medium friction factors, heat transfer coefficients, and effective solid thermal conductivity, which are described in Section 3.2. Further, by assuming that the engineering-scale effects of viscosity are accounted for with the porous media friction losses, viscous effects are neglected. Section 3.1 discusses the equations solved in Pronghorn for the SANA benchmark. 


\subsection{Governing Equations}

Pronghorn solves the porous media equivalents of the Euler equations for the fluid pressure $P$, momentum $\rho \vec{V}$, and temperature $T_{f}$, with several additional assumptions that are acceptable for nearly incompressible, slowly-evolving transients. The conservation of momentum equation is derived by averaging the Navier-Stokes conservation of momentum equation over a mixture of solid and fluid,

$$
\varepsilon \frac{\partial\left(\rho_{f} \vec{V}\right)}{\partial t}+\nabla \cdot\left(\varepsilon \rho_{f} \vec{V} \vec{V}\right)+\varepsilon \nabla P-\varepsilon \rho_{f} \vec{g}+W \rho_{f} \vec{V}=0
$$

where $\rho_{f}$ is the fluid intrinsic density, $\vec{V}$ the fluid intrinsic velocity, $P$ the fluid pressure, $\vec{g}$ the gravitational acceleration vector, and $W$ the porous medium friction coefficient. The second to fifth terms of this equation represents momentum advection, pressure gradient, gravity effect, and friction effect, respectively. Because it is assumed that the porosity is independent of time, the porosity is brought outside of the time derivative term. By neglecting the time rate of change of momentum and the advection of momentum, the momentum conservation equation simplifies to

$$
\varepsilon \nabla P-\varepsilon \rho_{f} \vec{g}+W \rho_{f} \vec{V}=0 .
$$

This form of the momentum equation is valid for low Reynolds number flows and slowlyevolving transients, since changes in momentum are instantaneously reflected as changes in pressure. This equation is solved for fluid momentum. The conservation of mass equation is derived by averaging the continuity equation over a mixture of solid and fluid in the same procedure as the momentum equation:

$$
\varepsilon \frac{\partial \rho_{f}}{\partial t}+\nabla \cdot\left(\varepsilon \rho_{f} \vec{V}\right)=0
$$

This purely advective equation can be transformed into a diffusive equation by rearranging Eq. (3) for momentum and substituting into the above equation,

$$
\varepsilon \frac{\partial \rho_{f}}{\partial t}+\nabla \cdot\left(\frac{\varepsilon}{W}\left[-\varepsilon \nabla P+\varepsilon \rho_{f} \vec{g}\right]\right)=0
$$

This pressure Poisson equation is solved for the fluid pressure because numerical solution to diffusive equations is easier than solution to the hyperbolic continuity equation, Eq. (4). The fluid density is provided by an equation of state. The fluid energy equation is derived by averaging the conservation of energy equation over a solid-fluid mixture in a manner identical to the mass and momentum equations. Equilibrium thermodynamics is used to transform an equation for the conserved total fluid energy to temperature. Compression work, viscous heating, and volumetric viscous heating are neglected, giving 


$$
\varepsilon \rho_{f} C_{p, f} \frac{\partial T_{f}}{\partial t}+\varepsilon \rho_{f} C_{p, f} \vec{V} \cdot \nabla T_{f}-\nabla \cdot\left(\varepsilon k_{f} \nabla T_{f}\right)+\alpha\left(T_{s}-T_{f}\right)=0,
$$

where $C_{p, f}$ is the fluid specific heat, $T_{f}$ the intrinsic fluid temperature, $k_{f}$ the fluid thermal conductivity, $\alpha$ the convective heat transfer coefficient, and $T_{s}$ the intrinsic solid temperature. This equation is solved for the fluid temperature. The second to fourth terms of this equation represents energy advection, heat conduction, and solid-to-fluid heat transfer, respectively. Because the heat source in the SANA experiments is not a volumetric source, and because the lack of fissile material means gamma ray energy deposition directly in the coolant is non-existent, no heat source is included in the fluid. The same derivation is followed for the solid energy equation, yielding

$$
(1-\varepsilon) \rho_{s} C_{p, s} \frac{\partial T_{s}}{\partial t}-\nabla \cdot\left(\kappa \nabla T_{s}\right)+\alpha\left(T_{f}-T_{s}\right)=0,
$$

where $\rho_{s}$ is the solid density, $C_{p, s}$ is the solid specific heat, and $\kappa$ is the effective solid thermal conductivity that accounts for various heat transfer modes between solid pebbles. These heat transfer modes include conduction within the pebbles and conduction within the fluid; conduction between pebbles at contact areas; and radiation heat transfer between pebbles. Similar to the fluid energy equation, because no direct heating of pebbles occurs in the SANA experiments, no heat source is included in the solid. In conclusion, the four equations solved by Pronghorn for the SANA benchmark are [4]:

- Eq. (5) for fluid pressure,

- Eq. (3) for fluid momentum,

- Eq. (6) for fluid temperature, and

- Eq. (7) for solid temperature.

No conservation of mass or momentum equations are solved for the solid because it is assumed that the solid phase is stationary. The following section discusses the constitutive relationships used for $\varepsilon, W, \alpha$, and $\kappa$, while Section 3.3 discusses the fluid and solid thermal properties used.

\subsection{Constitutive Relationships}

This section discusses the correlations used for the porous media constitutive terms $\varepsilon, W, \alpha$, and $\kappa$ in Pronghorn. Many researchers have developed experimental correlations for these terms, and this section only presents the forms used in the SANA benchmark validation.

A constant porosity is assumed throughout the bed for simplicity. The porosity in an infinite bed, $\varepsilon_{\infty}$, estimated by experimentalists typically ranges from 0.359 to 0.44 , depending on how the bed is filled [5]. Shaking a bed by vibration leads to relatively low $\varepsilon_{\infty}$, while draining fluid from 
a fluidized bed leads to relative high $\varepsilon_{\infty}$. For spheres dropped individually onto a bed that are then allowed to roll into place, $\varepsilon_{\infty}=0.4$. Because this is commonly how pebble bed reactors are fueled, $\varepsilon_{\infty}=0.4$ is selected for this benchmark [5]. Correlations are available in Pronghorn for exponentially-damped and exponentially-damped oscillatory porosity functions for future works.

The porous medium friction factor $W$ represents the sum of the Darcy and Forchheimer drag coefficients. The Darcy coefficient provides a momentum loss term that is linearly proportional to velocity, and hence represents friction drag. The proportionality of the Darcy friction coefficient can be derived for steady, unidirectional, laminar, and fully-developed flow. Friction drag, as opposed to form drag, dominates the total friction coefficient at low Reynolds numbers. At higher Reynolds numbers, boundary layers on the pebbles begin to separate, and form drag begins to dominate the friction coefficient. The Forchheimer drag coefficient provides a momentum loss term that is proportional to the square of velocity, and hence represents form drag. This Forchheimer drag component accounts for inertial drag effects. The Ergun correlation is commonly used for $W$, but overpredicts pressure drops at the higher Reynolds numbers typical of HTRs [6]. For helium, KTA provides an experimental correlation suitable for HTRs [7],

$$
W=\frac{160(1-\varepsilon)^{2}}{d^{2} \varepsilon} \frac{\mu_{f}}{\rho_{f}}+3\left(\frac{1-\varepsilon}{R e}\right)^{0.1} \frac{(1-\varepsilon)}{d}|\vec{V}|
$$

where $\mu_{f}$ is the fluid viscosity and $R e$ is the Reynolds number based on the extrinsic fluid velocity and pebble diameter. KTA also provides a correlation for the heat transfer coefficient in a bed of spherical pebbles that is used to determine the convective heat transfer coefficient [8],

$$
N u=1.27 \frac{\operatorname{Pr}^{1 / 3} \operatorname{Re}^{0.36}}{\varepsilon^{1.18}}+0.033 \frac{\operatorname{Pr}^{0.5} \operatorname{Re}^{0.86}}{\varepsilon^{1.07}},
$$

where $N u$ is the Nusselt number based on the pebble diameter and $\operatorname{Pr}$ the Prandtl number. To obtain the correct units for $\alpha$, the heat transfer coefficient computed by Eq. (9) must be multiplied by the ratio of the wetted heat transfer area to the heat transfer volume, $a_{w}$,

$$
\alpha=a_{w} \frac{k_{f} N u}{d}
$$

For a bed of spherical particles, $a_{w}$ is given as [2]

$$
a_{w}=\frac{6(1-\varepsilon)}{d}
$$

For heat transfer between the fluid and the bed wall, a second heat transfer coefficient is required. This heat transfer coefficient is utilized in the convection BC shown in Eq. (59), and represents convective heat transfer from the fluid to the bounding wall. For $50<\operatorname{Re}<2 \times 10^{4}$, Achenbach provides a correlation for the wall Nusselt number [2], 


$$
N u_{\text {wall }}=\left(1-\frac{d}{D}\right) \operatorname{Re}^{0.61} \operatorname{Pr}^{1 / 3}
$$

where $D$ is the bed diameter. The wall heat transfer coefficient $h_{\text {wall }}$ is defined as

$$
N u_{w a l l}=\frac{h_{w a l l} d}{k_{f}}
$$

$N u_{\text {wall }}$ represents heat transfer between the core coolant and the inner wall of the core vessel. Heat transfer also occurs by natural convection between the core vessel outer wall and the ambient environment. This heat transfer can be modeled using one of the many correlations for natural convection heat transfer from a flat plate, approximating the core vessel outer wall as a flat plate. In this report, several simplifications were introduced (discussed later), so an explicit modeling of solid wall (steel vessel) was not performed, and therefore this correlation was not directly used.

The effective solid thermal conductivity using in Pronghorn consists of three parallel heat transfer paths. These paths include radiation between pebbles, conduction facilitated by the fluid, and conduction in the pebbles, both within a single pebble and between pebbles at contact areas. $\kappa$ is the primary constitutive relationship that captures heat transfer mechanisms in loss of coolant accidents, and accurate correlations are essential to the prediction of decay heat removal in accidents without convective cooling. The overall $\kappa$ is the sum of these three heat transfer paths,

$$
\kappa=\kappa_{\text {radiation }}+\kappa_{\text {fluid conduction }}+\kappa_{\text {solid conduction }} \text {. }
$$

A modified Zehner, Bauer, and Schlünder model is used for $\kappa . \kappa$ is given as [9]

$$
\kappa=k_{f}(1-\sqrt{1-\varepsilon})\left(\frac{\varepsilon}{\varepsilon-1+1 / \mathscr{K}_{D}}+\varepsilon \mathscr{K}_{R}\right)+k_{f} \sqrt{1-\varepsilon}\left(\varphi \lambda+(1-\varphi) \mathscr{K}_{S F}\right)
$$

where $\varphi$ is the contact area fraction, or the ratio of the radius of the contact zone to the pebble radius. $\mathscr{K}_{D}$ represents a modified gas thermal conductivity that accounts for Knudsen effects when the mean free path of the fluid is on the order of the pebble diameter. When the mean free path is small, $\mathscr{K}_{D} \approx 1$. Because the mean free paths of helium and nitrogen are on the order of $\mu \mathrm{m}$ at SANA experiment conditions, this approximation is assumed throughout. $\mathscr{K}_{S F}$ is given by

$$
\begin{aligned}
\mathscr{K}_{S F}=\frac{2}{a} & {\left[\frac{\lambda+\mathscr{K}_{R}-1}{a^{2} \lambda \mathscr{K}_{D} / B} \ln \frac{\left(\lambda+\mathscr{K}_{R}\right) / B}{\left(\mathscr{K}_{D}+\left(1-\mathscr{K}_{D}\right)\left(\lambda+\mathscr{K}_{R}\right)\right)}-\frac{B-1}{a \mathscr{K}_{D}}\right.} \\
& \left.+\frac{B+1}{2 B}\left(\frac{\mathscr{K}_{R}}{\mathscr{K}_{D}}-B\left\{1+\frac{1-\mathscr{K}_{D}}{\mathscr{K}_{D}} \mathscr{K}_{R}\right\}\right)\right],
\end{aligned}
$$


where $\mathscr{K}_{R}$ represents the effective radiation thermal conductivity normalized to the fluid thermal conductivity. $B$ is given by

$$
B=C\left(\frac{1-\varepsilon}{\varepsilon}\right)^{m}
$$

For the case where Eq. (15) is simplified to represent only the fluid conduction component (by setting $\mathscr{K}_{R}=0$ and $\varphi=0$ ), Zehner and Schlünder recommend $C=1.25$ and $m=10 / 9$ to exactly fit the geometrical constraint on the shape factor $B$, but Hsu by a least squares procedure determined $C=1.364$ and $m=1.055$ better match the geometrical constraint. However, when all three components are considered, Hsu argues that the values for $C$ and $m$ in Eq. (17) should be modified [10]. Hsu provides graphical data for computing $C$ and $m$ as a function of the deformation ratio, but because the deformation does not change appreciably for pebble bed designs of practical interest, the constant values of $C=1.25$ and $m=10 / 9$ are used $[9,10]$. $a$ is defined as:

$$
a=\left[1+\frac{\mathscr{K}_{R}-B \mathscr{K}_{D}}{\lambda}\right] \frac{1}{\mathscr{K}_{D}}-B\left(\frac{1}{\mathscr{K}_{D}}-1\right)\left(1+\frac{\mathscr{K}_{R}}{\lambda}\right)
$$

The contact area fraction $\varphi$ has commonly been specified in the literature in terms of empirical values or graphical data that do not correlate well with the wide variety of porosities encountered $[9,10]$. You et. al. provide equations for $\varphi$ using elastic deformation theory, though $\varphi$ can only be approximated, since it is affected by the packing structure and surface roughness [9],

$$
\varphi=\frac{N_{c}}{4}\left(\frac{r_{c}}{r_{\text {pebble }}}\right)^{2}
$$

where $N_{c}$ is the average number of spheres in contact with each other (the coordination number). $N_{c}=6$ for simple cubic packings, $N_{c}=8$ for body centered cubic packings, and $N_{c}=12$ for face centered cubic packings. For randomly-packed pebble beds, the coordination number is some combination of these packing structures. You et. al calculate $N_{c}$ as a function of porosity as:

$$
N_{c}=\left(1.008 \times 10^{-2}\right) \varepsilon^{-4.785}+5.6480
$$

You estimates $10 \%$ accuracy of Eq. (20), though the equation provides slightly higher $N_{c}$ than actually observed [9]. Propagating this uncertainty to $\kappa_{\text {solid conduction }}$ results in only a $1 \%$ difference, and hence the error in Eq. (20) is acceptable. Then, Hertz deformation theory is used to calculate $R_{c} / R$ as

$$
\frac{r_{c}}{r_{\text {pebble }}}=\left(\frac{3}{4} \frac{1-v^{2}}{E} \frac{F}{r_{\text {pebble }}^{2}}\right)^{1 / 3}
$$


$v$ is the Poisson ratio of the solid, $E$ the Young's modulus of the solid, and $F$ the external force acting on the pebbles. $F$ represents the weight exerted on a pebble by all pebbles above, and is approximated using the infinite bed porosity $\varepsilon_{\infty}$ as

$$
F=\left(1-\varepsilon_{\infty}\right) \rho_{s} A_{c} z g_{z} \frac{S_{F}}{N_{A}}
$$

where $A_{c}$ is the bed cross-sectional area, $z$ the height above the pebble, $S_{F}$ a factor that correlates a force to a vertical component, and $N_{A}$ is the number of spheres per unit area. $N_{A}$ has different values depending on the assumed packing of the bed. For an average bed porosity of 0.4, a simple cubic packing provides the closest value of porosity that is not lower than the porosity. This choice leads to a conservative estimation of the contact heat transfer, since lower porosity yield increased solid conduction heat transfer fluxes. For a simple cubic packing, $N_{A}=1 / d^{2}[9]$.

Thus far, the radiation component represented by $\mathscr{K}_{R}$ has not been defined. The Breitbach and Barthels correlation provides a correction to the radiation proportionality shown in Eq. (15) to extend applicability to higher temperatures [11],

$$
\kappa_{\text {radiation }}=\frac{k_{s}}{\Lambda}\left[(1-\sqrt{1-\varepsilon}) \varepsilon+\frac{\sqrt{1-\varepsilon}}{2 / \varepsilon_{s}-1} \frac{B+1}{B} \frac{1}{1+\frac{1}{\left(2 / \varepsilon_{s}-1\right) \Lambda}}\right]
$$

where $\sigma$ is the Stefan-Boltzmann constant, $\varepsilon_{s}$ the solid emmissivity, and $\Lambda$ is given as

$$
\Lambda=\frac{k_{s}}{4 \sigma T_{s}^{3} d}
$$

Therefore, the modified Zehner, Bauer, and Schlünder correlation used in Pronghorn is Eq. (15) with $\mathscr{K}_{R}=0$, and with terms in this correlation defined in Eqs. (16)-(22). The radiation component is provided separately by Eq. (23) by eliminating the radiation component in Eq. (15).

For comparison, a second formulation for $\kappa$ is also investigated. This alternative formulation

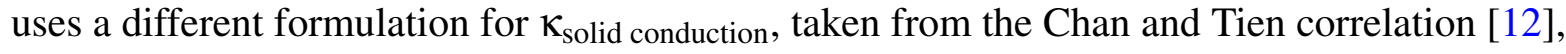

$$
\kappa_{\text {solid conduction }}=\frac{k_{s}}{0.53} \frac{N_{A}}{N_{L}}\left(\frac{3}{8} \frac{1-v^{2}}{E} F d_{\text {pebble }}\right)^{1 / 3}
$$

where $F$ is given by Eq. (22) and $N_{L}$ is the number of pebbles per unit length, which depends on the assumed packing fraction. Eq. (15) with $\mathscr{K}_{R}=0, \mathscr{K}_{D}=1$, and $\varphi=0$ is used in combination with Eqs. (23) and (25) to replicate the formulation used for $\kappa$ in THERMIX [13].

To validate the correctness of the Zehner-Bauer-Schlünder correlation implemented in Pronghorn, a special simulation is performed with the following conditions. The pebble bed and helium gas 
are artificially kept in nearly thermal equilibrium conditions, the pressure held at atmospheric pressure, and the pebble bed temperature varying from $100 \mathrm{~K}$ to $1500 \mathrm{~K}$. In figure 2, the Zehner-BauerSchlünder correlation $\kappa$ is plotted against SANA experimental measurements and the comparison values calculated by the THERMIX code methodology discussed above [13]. Both correlations predict smaller $\kappa$ because the measurements for $\kappa$ performed by the SANA experimentalists cannot fully correct for fluid convection effects. In both Pronghorn and THERMIX, convection is modeled by a convective term in the fluid energy equation, rather than by incorporating convection physics into $\kappa$, and hence this underprediction is to be expected. At higher temperatures, the Zehner-Bauer-Schlünder correlation agrees slightly better with the SANA measurements, and hence is used throughout the remainder of this report.

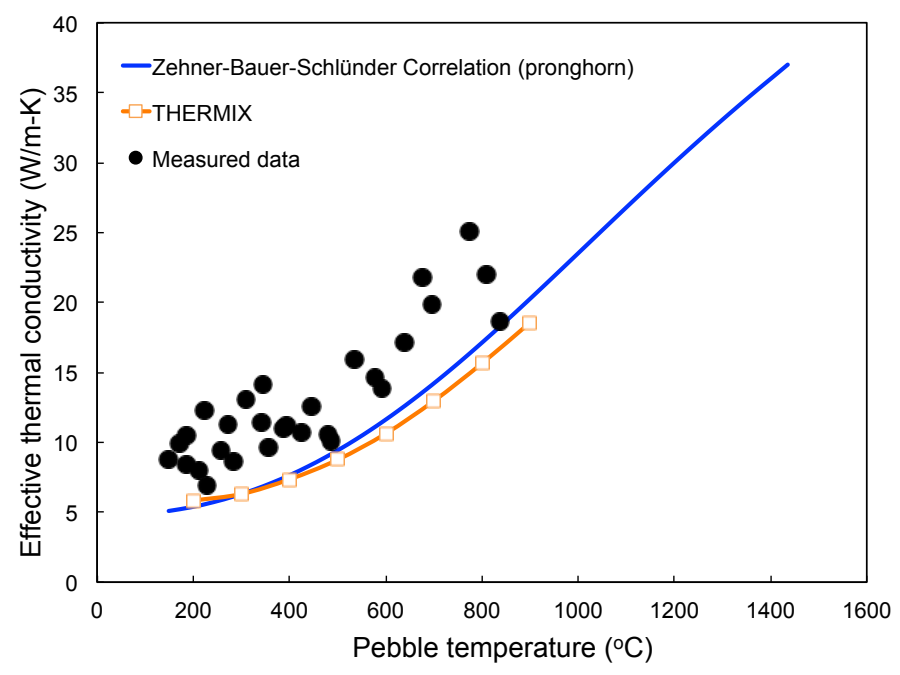

Figure 2. Validation of Zehner-Bauer-Schlünder correlation (pronghorn) predicted effective thermal conductivity values compared to experimental measurements and THERMIX predicted values. [13]

\subsection{Thermo-Physical Properties}

In addition to $\varepsilon, W, \alpha$, and $\kappa$, constitutive relationships are required for fluid and solid thermophysical properties. Many codes developed for HTR analysis assume the ideal gas equation of state. Pronghorn permits considerable flexibility in defining thermophysical properties, as any equation of state available in the Multiphysics Object-Oriented Simulation Environment (MOOSE) fluid properties module, or a custom set of fluid properties, may be used. This is an important improvement upon earlier codes, as Pronghorn is intended for application to liquid-cooled pebble bed reactors as well, where the ideal gas law approximation produces greater errors.

Fluid properties required by the governing equations include isobaric specific heat, density, viscosity, and thermal conductivity. Correlations for helium density, viscosity, and thermal conductivity, and a constant value for isobaric specific heat of $5195 \mathrm{~J} / \mathrm{kg} \cdot \mathrm{K}$, are obtained from [14]. These correlations are valid in the range $1 \leq P \leq 100$ bar and $273 \leq T_{f} \leq 1800 \mathrm{~K}$, and have been 
used by KTA in their analysis of HTRs [15]. Density, viscosity, and thermal conductivity are given as:

$$
\begin{gathered}
\rho_{f}\left(\mathrm{~kg} / \mathrm{m}^{3}\right)=48.14 \frac{P}{T_{f}}\left(1+0.4446 \frac{P}{T_{f}^{1.2}}\right)^{-1}, \\
\mu_{f}(\mathrm{~Pa} \cdot \mathrm{s})=\left(3.674 \times 10^{-7}\right) T_{f}^{0.7}, \\
k_{f}(\mathrm{~W} / \mathrm{m} \cdot \mathrm{K})=\left(2.682 \times 10^{-3}\right)\left(1+\left(1.123 \times 10^{-3}\right) P\right) T_{f}^{0.71\left(1-\left(2 \times 10^{-4}\right) P\right)},
\end{gathered}
$$

where $P$ is given in bar and $T_{f}$ in $\mathrm{K}$. Because helium is a noble gas, there is not a significant difference in these thermal properties from those obtained with the ideal gas law, so the ideal gas law would also be appropriate. For the same reason, the ideal gas law equation of state is used for the nitrogen gas density,

$$
\rho_{f}\left(\mathrm{~kg} / \mathrm{m}^{3}\right)=\frac{P M_{N_{2}}}{R T_{f}}
$$

where $P$ is given in $\mathrm{Pa}$ and $T_{f}$ in $\mathrm{K} . R=8.3144598 \mathrm{~J} / \mathrm{mol} \cdot \mathrm{K}$ is the gas constant. The molar weight of the nitrogen gas is $M_{N_{2}}=28.0134 \times 10^{-3} \mathrm{~kg} / \mathrm{mol}$. Other thermal properties are provided as constants for simplicity. For nitrogen gas, viscosity is given as $\mu_{f}=3.5932 \times 10^{-5} \mathrm{~Pa} \cdot \mathrm{s}$, thermal conductivity as $k_{f}=0.055197 \mathrm{~W} / \mathrm{m} \cdot \mathrm{K}$, and isobaric specific heat as $1122.3 \mathrm{~J} / \mathrm{kg} \cdot \mathrm{K}$. These constant values are evaluated at $\mathrm{P}=1$ atmospheric pressure and temperature $T_{f}=800 \mathrm{~K}$ based on the National Institute of Standards and Technology (NIST) standard [16]. While density is not assumed constant, selecting constant viscosity and thermal conductivity will introduce nonnegligible errors in the simulation results due to the large temperature gradients in the SANA experiments. The inclusion of a more sophisticated thermal property library for nitrogen and other fluids is scheduled to be implemented in MOOSE at the framework level, and therefore we do not intend to reproduce such an effort in this work.

Solid properties required by the governing equations include isobaric specific heat, density, and thermal conductivity. The emissivity, Young's modulus, and Poisson ratio are also required for evaluating $\kappa$. It is a common approximation that solid properties are independent of pressure, so none of the correlations used in this benchmarking effort are functions of pressure. For the electric graphite pebbles, SANA provides tabular data for thermal conductivity [1]. The data from "Measurement 3" is used, which gives a constant density as $\rho_{s}=1673 \mathrm{~kg} / \mathrm{m}^{3}$. The tabular thermal conductivity data is fit as a function of temperature as

$$
k_{s}(\mathrm{~W} / \mathrm{m} \cdot \mathrm{K})=\left(2.5738 \times 10^{4}\right) T_{s}^{-0.86367},
$$


where $T_{s}$ is given in K. SANA report [1] does not provide data for solid isobaric specific heat. Butland collected isobaric specific heat data from a variety of sources for many types of graphite, none actually including nuclear graphite, and then attempted to aggregate the data sets that best represent nuclear graphite into a single correlation [17],

$$
\begin{aligned}
C_{p, s}(\mathrm{~J} / \mathrm{kg} \cdot \mathrm{K})=4184 & \left(0.54212-2.42667 \times 10^{-6} T_{s}-\frac{90.2725}{T_{s}}\right. \\
& \left.-\frac{43449.3}{T_{s}^{2}}+\frac{1.59309 \times 10^{7}}{T_{s}^{3}}-\frac{1.43688 \times 10^{9}}{T_{s}^{4}}\right),
\end{aligned}
$$

where $T_{s}$ is given in $\mathrm{K}$. 


\section{Numerical Method}

This section presents a very high-level description of the numerical method used to solve Eqs. (3), (5), (6), and (7). Pronghorn solves these equations using the Bubnov-Galerkin finite element method. By multiplying the governing equations by a test function $\psi$ and integrating over all space, the weak forms are obtained,

$$
\begin{array}{r}
\left(\varepsilon \frac{\partial \rho_{f}}{\partial t}, \psi\right)+\left(-\frac{\varepsilon}{W}\left[-\varepsilon \nabla P+\varepsilon \rho_{f} \vec{g}\right], \nabla \psi\right)+\left\langle\frac{\varepsilon}{W}\left[-\varepsilon \nabla P+\varepsilon \rho_{f} \vec{g}\right], \psi\right\rangle=0 \\
\left(\varepsilon \frac{\partial \rho_{f} V_{i}}{\partial t}-\varepsilon \rho_{f} g_{i}+W \rho_{f} V_{i}+\varepsilon \nabla P, \psi\right)=0 \\
\left(\varepsilon \rho_{f} C_{p, f} \frac{\partial T_{f}}{\partial t}-\varepsilon \rho_{f} C_{p, f} \vec{V} \cdot \nabla T_{f}+\alpha\left(T_{f}-T_{s}\right), \psi\right)+\left(\varepsilon k_{f} \nabla T_{f}, \nabla \psi\right)+<-\varepsilon k_{f} \nabla T_{f}, \psi>=0 \\
\left((1-\varepsilon) \rho_{s} C_{p, s} \frac{\partial T_{s}}{\partial t}+\alpha\left(T_{s}-T_{f}\right), \psi\right)+\left(\kappa_{s} \nabla T_{s}, \nabla \psi\right)-<\kappa_{s} \nabla T_{s}, \psi>=0
\end{array}
$$

where $i$ refers to the $i$-th momentum equation. In writing the weak forms, the following notation is introduced for a volumetric integral,

$$
\int_{\Omega} f \psi d \Omega \equiv(f, \psi)
$$

and for a surface integral,

$$
\int_{\partial \Omega} f \psi \cdot \vec{n} d \Omega \equiv\langle f, \psi\rangle
$$

where $f$ is a generic function, $\Omega$ is a volume, $\partial \Omega$ is a surface, and $\vec{n}$ is the unit normal vector of the surface. The BCs in this equation are described in Section 4.1. The weak forms given in Eq. (32) together represent the equation system

$$
\vec{F}(\vec{u})=0
$$

where $\vec{F}$ represents the residual vector and $\vec{u}$ the numerical solution. For the equations shown in Eq. (32), $\vec{F}$ is:

$$
\vec{F}=\left[\begin{array}{llllll}
\vec{F}_{p} & \vec{F}_{m, 1} & \vec{F}_{m, 2} & \vec{F}_{m, 3} & \vec{F}_{f} & \vec{F}_{s}
\end{array}\right]^{T}
$$

where the components of $\vec{F}$ are defined as 


$$
\begin{aligned}
\vec{F}_{p}(P)= & \left(\varepsilon \frac{\partial \rho_{f}}{\partial t}, \psi\right)+\left(-\frac{\varepsilon}{W}\left[-\varepsilon \nabla P+\varepsilon \rho_{f} \vec{g}\right], \nabla \psi\right)+\left\langle\frac{\varepsilon}{W}\left[-\varepsilon \nabla P+\varepsilon \rho_{f} \vec{g}\right], \psi\right\rangle \\
\vec{F}_{m, i}\left(\rho_{f} \vec{V}\right)= & \left(\varepsilon \frac{\partial \rho_{f} V_{i}}{\partial t}-\varepsilon \rho_{f} g_{i}+W \rho_{f} V_{i}+\varepsilon \nabla P, \psi\right) \\
\vec{F}_{f}\left(T_{f}\right)= & \left(\varepsilon \rho_{f} C_{p, f} \frac{\partial T_{f}}{\partial t}-\varepsilon \rho_{f} C_{p, f} \vec{V} \cdot \nabla T_{f}+\alpha\left(T_{f}-T_{s}\right), \psi\right)+\left(\varepsilon k_{f} \nabla T_{f}, \nabla \psi\right) \\
& +<-\varepsilon k_{f} \nabla T_{f}, \psi> \\
\vec{F}_{s}\left(T_{s}\right)= & \left((1-\varepsilon) \rho_{s} C_{p, s} \frac{\partial T_{s}}{\partial t}+\alpha\left(T_{s}-T_{f}\right), \psi\right)+\left(\kappa_{s} \nabla T_{s}, \nabla \psi\right)-\left\langle\kappa_{s} \nabla T_{s}, \psi\right\rangle .
\end{aligned}
$$

The subscripts $p, m, f$, and $s$ refer to the pressure, momentum, fluid energy, and solid energy equations, respectively. The solution vector $\vec{u}$ is a single vector containing all the problem unknowns. For instance, pressure, momentum, fluid temperature, and solid temperature are solved for at each node in Pronghorn. The solution vector therefore is of the form

$$
\begin{aligned}
& \vec{u}=\left[\rho_{f, 1} \quad \rho_{f, 1} V_{x, 1} \quad \rho_{f, 1} V_{y, 1} \quad \rho_{f, 1} V_{z, 1} \quad T_{f, 1} \quad T_{s, 1} \quad \cdots\right. \\
& \left.\rho_{f, n} \quad \rho_{f, n} V_{x, n} \quad \rho_{f, n} V_{y, n} \quad \rho_{f, n} V_{z, n} \quad T_{f, n} \quad T_{s, n}\right]^{T},
\end{aligned}
$$

where the numbered subscripts denote node numbers $1 \leq n \leq N$ and $N$ is the total number of nodes. The finite element method solves Eq. (35) by assuming the numerical solution $\vec{u}$ lies in the same space as the test function $\psi$. Both are expressed as a linear sum of shape functions $\phi$ multiplied by expansion coefficients $u_{j}$. The expansion coefficients for the test function are unity. These functions may be Lagrange, Hermite, monomial, or any of the other shape functions available in MOOSE.

$$
\vec{u}=\sum_{j=1}^{N} u_{j} \phi_{j}
$$

Hence, the entries in the numerical solution vector in Eq. (38) represents the expansion coefficients from Eq. (39) at the particular node. The solution is reconstructed via Eq. (39) to a continuous field after the numerical method in MOOSE has solved Eq. (35) to an acceptable tolerance.

As discussed, the Finite Element Method (FEM) is used to discretize the weak forms in Eq. (32) to provide a discretized set of nonlinear equations represented by the general form in Eq. (35). Traditional linear algebra techniques cannot be used to directly solve Eq. (35) because it is nonlinear. Therefore, MOOSE linearizes Eq. (35) into the form

$$
\mathbf{A} \vec{x}=\vec{b}
$$


where $\mathbf{A}$ is an $N \times N$ matrix, $\vec{x}$ is the $N \times 1$ linear solution vector, and $b$ is the $N \times 1$ right-handside vector. Once in a linear form, any number of linear algebra solution techniques may be used to solve Eq. (40). So, the numerical method in MOOSE consists of (at least) two iteration loops -

1. An outer loop over nonlinear iterations that compute better and better guesses to $\vec{u}$ in Eq. (35), and

2. an inner loop over linear iterations that compute better and better guesses to $\vec{x}$ in Eq. (40).

Once the linear solution method has computed $\vec{x}$ that sufficiently solves Eq. (40), that $\vec{x}$ is used in a simple update formula to compute the updated $\vec{u}$. $i$ is used for the outer loop iteration index, and $k$ for the inner loop index for clarity in the remainder of this section. MOOSE solves nonlinear equations using the Jacobian-Free Newton-Krylov (JFNK) method, which is a combination of a Newton method for the outer iterations and a Generalized Minimum Residual Method (GMRES) Krylov subspace method for the inner iterations. A brief description of the Newton method linearization and the GMRES Krylov subspace method used for the linear iterations is provided, with more detailed descriptions available in the literature [18].

Newton's method forms a linear approximation (about the current iterate $\vec{u}_{i}$ ) to the root-finding problem in Eq. (35) by forming the Taylor series expansion for the next solution iterate,

$$
\vec{F}\left(\vec{u}_{i+1}\right)=\vec{F}\left(\vec{u}_{i}\right)+\frac{\partial \vec{F}\left(\vec{u}_{i}\right)}{\partial \vec{u}}\left(\vec{u}_{i+1}-\vec{u}_{i}\right)+\mathscr{O}\left(\left(\vec{u}_{i+1}-\vec{u}_{i}\right)^{2}\right)=0
$$

By neglecting quadratic and higher-order terms, an iterative update formula results,

$$
\begin{aligned}
\frac{\partial \vec{F}\left(\vec{u}_{i}\right)}{\partial \vec{u}}\left(\vec{u}_{i+1}-\vec{u}_{i}\right) & =-\vec{F}\left(\vec{u}_{i}\right) \\
\mathbf{J}\left(\vec{u}_{i}\right) \vec{\delta}_{i} & =-\vec{F}\left(\vec{u}_{i}\right) .
\end{aligned}
$$

This linear equation is of the form $\mathbf{A} \vec{x}=\vec{b}$, and is solved for $\vec{\delta}_{i} \equiv \vec{u}_{i+1}-\vec{u}_{i}$ using a linear solution method (in this case, GMRES). In other words, in the syntax defined for the linear solution method, $\vec{\delta}_{i}$ represents $\vec{x}_{k}$ in $\mathbf{A} \vec{x}_{k}=\vec{b}$. The Jacobian is defined as

$$
\mathbf{J}\left(\vec{u}_{i}\right)=\frac{\partial \vec{F}\left(\vec{u}_{i}\right)}{\partial \vec{u}}
$$

The GMRES linear solution method is then used to solve Eq. (42) for $\vec{\delta}_{i}$, and a simple update is performed to provide the next solution iterate,

$$
\vec{u}_{i+1}=\vec{u}_{i}+\vec{\delta}_{i}
$$


The GMRES is a Krylov subspace method. A Krylov space is a vector space built by repeatedly applying a matrix to an initial vector. The residual $\vec{r}$ is defined as the difference in the true $\vec{b}$ and the numerical approximation to $\vec{b}$,

$$
\vec{r}_{k}=\vec{b}-\mathbf{A} \vec{x}_{k}
$$

The GMRES method seeks an optimal approximation to $\vec{x}_{k+1}$ for general non-symmetric systems of the form Eq. (40) from the Krylov subspace $\mathscr{D}_{k}$ of dimension $k$,

$$
\mathscr{D}_{k}=\operatorname{span}\left\{\vec{r}_{0}, \mathbf{A} \vec{r}_{0}, \cdots, \mathbf{A}^{k-1} \vec{r}_{0}\right\}
$$

In every iteration, $\vec{x}_{k+1}$ is determined by solving a least-squares minimization problem. To solve this minimization problem, an orthonormal basis for $\mathscr{D}_{k}$ is required. This orthonormal basis is denoted as $\mathbf{Q}_{k}$,

$$
\mathbf{Q}_{k}=\left[\begin{array}{llll}
\vec{q}_{1} & \vec{q}_{2} & \cdots & \vec{q}_{k}
\end{array}\right]^{T},
$$

where each column of $\mathbf{Q}_{k}$ is an orthonormal vector $\vec{q}_{k}$ of length $N$, and $N$ of these vectors are required to establish a complete basis for $\mathscr{D}_{k}$. The vectors in $\mathbf{Q}_{k}$ can be determined via the Arnoldi process.

Each iteration of the Arnoldi process chooses some new linearly independent vector $\vec{s}$ and orthonormalizes it to $\mathscr{D}_{k}$ using a Gram-Schmidt procedure. A natural choice would be simply to compute the next vector in the Krylov space, $\mathbf{A}^{k} \vec{r}_{0}$. However, as $k$ becomes large, the vectors in $\mathscr{D}_{k}$ become increasingly linearly dependent because formation of the Krylov subspace in this manner is equivalent to power iteration. The starting vector $\vec{s}_{k+1}$ for computing $\vec{q}_{k+1}$ is selected as $\vec{s}_{k+1}=\mathbf{A} \vec{q}_{k}$. For each iteration, $\vec{s}_{k+1}$ is orthogonalized via a Gram-Schmidt procedure against all previous vectors in $\mathbf{Q}_{k}$; after orthogonalization, $\vec{s}_{k+1}$ is normalized to a unit vector. For each iteration, the Arnoldi process adds one vector, $\vec{q}_{k+1}$ to $\mathbf{Q}_{k}$ by the following procedure.

$$
\begin{aligned}
& \vec{s}_{k+1}=\mathbf{A} \vec{q}_{k} \\
& \vec{s}_{k+1}=\vec{s}_{k+1}-\underbrace{\vec{q}_{i}^{T} \vec{s}_{k+1}}_{h_{i k}} \vec{q}_{i}, \text { for } i<k+1 \quad \text { (orthogonalization) } \\
& \vec{q}_{k+1}=\vec{s}_{k+1} /\left\|\vec{s}_{k+1}\right\|_{2} \quad \text { (normalization). }
\end{aligned}
$$

From the above algorithm, a matrix of size $k+1 \times k$ can be defined, 


$$
\tilde{\mathbf{H}}_{k}=\left[\begin{array}{ccccc}
h_{11} & h_{12} & h_{13} & \cdots & h_{1 k} \\
\left\|\vec{s}_{2}\right\|_{2} & h_{22} & h_{23} & \cdots & h_{2 k} \\
0 & \left\|\vec{s}_{3}\right\|_{2} & h_{33} & \cdots & h_{3 k} \\
0 & 0 & \left\|\vec{s}_{4}\right\| & \ddots & h_{4 k} \\
0 & 0 & 0 & \cdots & \left\|\vec{s}_{k+1}\right\|_{2}
\end{array}\right]
$$

In this form,

$$
\begin{aligned}
\mathbf{A} \mathbf{Q}_{k} & =\mathbf{Q}_{k} \mathbf{H}_{k}+\vec{s}_{k+1} \vec{e}_{k}^{T} \\
& =\mathbf{Q}_{k+1} \tilde{\mathbf{H}}_{k},
\end{aligned}
$$

where $\vec{e}_{k}=\left[\begin{array}{llll}0 & 0 & \cdots & 1\end{array}\right]^{T}$. If the Arnoldi process is performed for $k=N$ iterations, then in the $N$-th iteration, $\mathbf{A} \vec{q}_{N}$ will lie in $\mathscr{D}_{N}$ because the basis $\mathbf{Q}_{N}$ is already all of $\mathbb{R}^{N}$. For $N$ iterations, the Arnoldi process produces a similarity transformation of $\mathbf{A}$ to upper Hessenberg form. However, the goal of iterative methods is to converge in substantially less than $N$ iterations.

After some smaller number of iterations, the GMRES algorithm computes the next iterate $\vec{x}_{k+1}$ from the space $\vec{x}_{0}+\mathscr{D}_{k}$ by minimizing the L-2 norm of the residual over $\vec{x}_{0}+\mathscr{D}_{k}$. Because the vectors in $\mathbf{Q}_{k}$ form a basis for $\mathscr{D}_{k}$, we can instead specify that $\vec{x}_{k+1}$ be in:

$$
\vec{x}_{k+1}=\vec{x}_{0}+\mathbf{Q}_{k} \vec{y}_{k}
$$

for some vector $\vec{y}_{k} \in \mathbb{R}^{k}$. The residual is:

$$
\begin{aligned}
\vec{r}_{k+1} & =b-\mathbf{A}\left(\vec{x}_{0}+\mathbf{Q}_{k} \vec{y}_{k}\right) \\
& =\vec{r}_{0}-\mathbf{A} \mathbf{Q}_{k} \vec{y}_{k} \\
& =\vec{r}_{0}-\mathbf{Q}_{k+1} \tilde{\mathbf{H}}_{k} \vec{y}_{k}
\end{aligned}
$$

where Eq. (50) is used. Because the first column in $\mathbf{Q}_{k+1}$ is simply the starting point of the Arnoldi process, $\vec{r}_{0} /\left\|\vec{r}_{0}\right\|_{2}$, the residual can be expressed as:

$$
\vec{r}_{0}=\mathbf{Q}_{k+1} \vec{\eta}
$$

where $\vec{\eta}=\left[\begin{array}{llll}\left\|r_{0}\right\|_{2} & 0 & \cdots & 0\end{array}\right]^{T}$. So, $\left\|\vec{r}_{k+1}\right\|_{2}$ becomes:

$$
\left\|\vec{r}_{k+1}\right\|_{2}=\left\|\vec{\eta}-\tilde{\mathbf{H}}_{k} \vec{y}_{k}\right\|_{2}
$$


where $\mathbf{Q}_{k+1}^{T} \mathbf{Q}_{k+1}=\mathbf{I}$. So, in each iteration of GMRES, the next iterate solution is obtained by minimization of the L-2 norm of the residual by solving a least squares problem for $\vec{y}_{k}$, and then using Eq. (51) to solve for the next iterate $\vec{x}_{k+1}$ :

$$
\min _{\vec{y}_{k} \in \mathbb{R}^{k}}\left\|\eta-\tilde{\mathbf{H}}_{k} \vec{y}_{k}\right\|_{2}
$$

In each linear iteration, the GMRES algorithm performs a least squares minimization of the L-2 norm of the residual over the basis space built up by the Arnoldi process. When a sufficient number of iterations have been performed such that the residual in Eq. (45) is sufficiently small, the next Newton iteration is performed. This nonlinear-linear iteration procedure is completed until the numerical solution provides a residual in Eq. (35) that is sufficiently small.

This combination is referred to as a "Newton-Krylov" method, though this name is often used in the literature to imply one extra caveat - that the Jacobian is never explicitly formed. $\mathbf{J}\left(\vec{u}_{i}\right) \delta_{i}$ in Eq. (42) simply represents the directional derivative of $\vec{F}\left(\vec{u}_{i}\right)$ in the direction of the vector $\vec{\delta}_{i}$. While the Jacobian holds directional derivative information for all directions, the goal of iterative methods is to perform searches in a small number of directions such that the directional derivative information is only needed for a few of these directions such that formation of the Jacobian is not needed. "Newton-Krylov" methods, or JFNK methods, approximate the action of the Jacobian on the vector $\vec{\delta}_{i}$ using a first-order Finite Difference (FD) derivative:

$$
\mathbf{J}\left(\vec{u}_{i}\right) \approx \frac{\vec{F}\left(\vec{u}_{i}+\varepsilon \vec{\delta}_{i}\right)-\vec{F}\left(\vec{u}_{i}\right)}{\varepsilon}
$$

where $\varepsilon$ is a small number, typically chosen as $\varepsilon=\sqrt{\varepsilon_{\text {mach }}}$, where $\varepsilon_{\text {mach }}$ is the machine precision, to balance accuracy of the FD approximation with potential overflow from division by a small number.

\subsection{Boundary Conditions}

Two general types of BCs, Dirichlet and Neumann, can be specified in Pronghorn. A Dirichlet BC simply sets a known value for a variable on a boundary. This type of BC is strongly enforced, meaning the variable on that boundary is exactly equal to the known Dirichlet value. Neumann BCs set a known value for an expression that is not identical to one of the solution variables. For example, a Neumann condition is needed to specify a heat flux on a boundary, since a Dirichlet condition cannot be used to assign a value to the heat flux because temperature, rather than heat flux, is the solution variable of the temperature equation. Neumann conditions are therefore only weakly enforced.

For the weak forms in Eq. (32), the terms with angled brackets whose notation is defined in Eq. (34) represent the available Neumann-type conditions for each equation. A Neumann condition 
must be specified if a Dirichlet condition is not specified. In the remainder of this section, the available boundary conditions for each equation are discussed. In Section 5, the particular BCs used for each experiment in Table 2 will be described. In the remainder of this section, $i$ subscripts denote known inlet values, $o$ subscripts denote known outlet values, and a tilde over a variable, $\tilde{q}$, denotes a known value of the variable, $q$, at any boundary.

The Neumann-type BCs for the mass equation arise by recognizing that the advective term in the pressure Poisson equation is the same as the advective term in the conservation of mass equation,

$$
\frac{\varepsilon}{W}\left[-\varepsilon \nabla P+\varepsilon \rho_{f} \vec{g}\right] \cdot \vec{n}= \begin{cases}\rho_{f, i} \vec{V}_{i} \cdot \vec{n} & \text { on } \partial \Omega_{\text {in }} \\ \rho_{f} \vec{V} \cdot \vec{n} & \text { on } \partial \Omega_{\text {out }} \\ 0 & \text { on } \partial \Omega_{\text {wall }}\end{cases}
$$

For advective equations, because information travels along characteristics that cannot travel against the direction of velocity, a Neumann-type $\mathrm{BCn}$ that sets a value for the momentum can only be specified on inflow boundaries. Because the default boundary condition that arises from the finite element method is the "no-boundary" condition, a special outflow boundary condition is required for outflow boundaries simply to avoid a zero flux boundary condition. The no-slip condition at the wall applies a zero normal velocity component. Because the Pressure Poisson equation is not purely advective due to the Laplacian of pressure, a Dirichlet condition for pressure can be specified on any boundary, provided a Neumann condition is not also specified on that boundary. This Dirichlet boundary is often selected as the outflow boundary, since it is desirable to still be able to specify an inlet momentum on the inflow boundary and no-slip on the walls. Therefore, the combined Neumann and Dirichlet boundary conditions used in the pressure Poisson equation in Pronghorn are

$$
\begin{aligned}
\frac{\varepsilon}{W}\left[-\varepsilon \nabla P+\varepsilon \rho_{f} \vec{g}\right] \cdot \vec{n} & = \begin{cases}\rho_{f, i} \vec{V}_{i} \cdot \vec{n} & \text { on } \partial \Omega_{\text {in }} \\
0 & \text { on } \partial \Omega_{\text {wall }}\end{cases} \\
P & =P_{o} \quad \text { on } \partial \Omega_{\text {out }} .
\end{aligned}
$$

Because integration by parts is not used on any terms in the momentum equation, no BCs are needed for the momentum equation directly. The inlet value for momentum is specified via the inlet Neumann condition in the pressure Poisson equation.

The Neumann-type BCs for the fluid energy equation include the specification of a known heat flux value or a convective cooling heat flux with the ambient wall temperature,

$$
-\varepsilon k_{f} \nabla T_{f} \cdot \vec{n}= \begin{cases}\tilde{q}_{f} & \partial \Omega_{\text {known heat flux }} \\ h_{\text {wall }}\left(T_{f}-T_{\text {wall }}\right) & \partial \Omega_{\text {convection }}\end{cases}
$$

where $\tilde{q}_{f}$ is a known value of the heat flux and $T_{\text {wall }}$ is the wall ambient temperature. For 
insulated boundaries, the known heat flux is zero. Radiation heat transfer between the fluid and the surroundings is neglected, as the solid temperature for reactor applications will be significantly larger than the fluid temperature. A further simplification is made to assume that, near the outer wall region of the SANA experiment, the fluid transfers its heat to the solid pebbles first, and then the solid structure loses heat to the ambient via both natural convection heat transfer and thermal radiation heat transfer. Under this assumption, the value of $h_{\text {wall }}$ is set to be zero in equation (59).

The Neumann-type BCs for the solid energy equation are similar to those for the fluid energy equation, except that a thermal radiation $\mathrm{BC}$ is used in place of the convective cooling $\mathrm{BC}$,

$$
-\kappa \nabla T_{s} \cdot \vec{n}= \begin{cases}\tilde{q}_{s} & \partial \Omega_{\text {known heat flux }} \\ \varepsilon \sigma\left(T_{s}^{4}-T_{\infty}^{4}\right)+h_{N C}\left(T_{s}-T_{\infty}\right) & \partial \Omega_{\text {radiation }}\end{cases}
$$

where $\tilde{q}_{s}$ is a known value of the heat flux, $\varepsilon$ is the emissivity of the solid pebbles, $h_{N C}$ the natural convection heat transfer at the outer wall, and $T_{\infty}$ the ambient temperature. For insulated boundaries, the known heat flux is zero. 


\section{Modeling Assumptions}

This section presents the assumptions made in generating the Pronghorn input files for the experiments listed in Table 2. The assumptions made in the governing equations, constitutive models, and thermophysical properties have already been described in Sections 3.1, 3.2, and 3.3, respectively. Therefore, this section presents the additional interpretations of the SANA benchmark specifications and translation to Pronghorn input files.

\subsection{Cylindrical Geometry}

Because the asymmetries in the SANA benchmark due to thermocouple and cooling equipment placement is expected to have a negligible on simulation results, the geometry is approximated as a 2-D $r-z$ cylindrical geometry that is only a function of the radial coordinate $r$ and axial coordinate $z$. Many existing porous media HTR codes make this assumption, but it should be noted that Pronghorn can use 3-D unstructured meshes as well [13,19].

MOOSE is dimension-agnostic, but because the divergence operator is different in cylindrical and Cartesian coordinate systems, several additional terms are provided in the weak forms of kernels with a divergence operator in the strong form. In 2-D Cartesian coordinates, the divergence operator acting on a vector field $\vec{f}$ is

$$
\nabla \cdot \vec{f}=\frac{\partial f_{x}}{\partial x}+\frac{\partial f_{y}}{\partial y}
$$

while in cylindrical coordinates, the divergence operator is

$$
\begin{aligned}
\nabla \cdot \vec{f} & =\frac{1}{r} \frac{\partial}{\partial r}\left(r f_{r}\right)+\frac{\partial f_{z}}{\partial z} \\
& =\underbrace{\frac{f_{r}}{r}}_{\text {new term }}+\frac{\partial f_{r}}{\partial r}+\frac{\partial f_{z}}{\partial z}
\end{aligned}
$$

Provided the geometry is set up such that the $x$-coordinate corresponds to the radial direction and the $z$-coordinate corresponds to the axial direction, then to run in $r$ - $z$ geometry simply requires the inclusion of the additional "new term" shown in Eq. (62). These additional terms are included for the divergence terms in the pressure, fluid energy, and solid energy equations, allowing the SANA benchmark to be approximated as an azimuthally-symmetric cylindrical geometry. 


\subsection{Geometry}

The SANA benchmark specifications include geometrical information for the packed bed, thermocouples, thermocouple casings, and insulation layers on the top and bottom of the bed. Only the packed bed is modeled in Pronghorn, such that boundary conditions are specified at the walls of the packed bed and at the outer surface of the central heating element. The insulation layers and thermocouple casings are not modeled explicitly. The thermocouple casings are neglected in the model, while the effects of insulation layers are accounted for by the choices of BCs discussed in Section 5.3. Therefore, the mesh used is a 2-D rectangular-element mesh of height $1 \mathrm{~m}$ and width $0.6795 \mathrm{~m}$.

\subsection{Boundary Conditions}

This section presents the choices of and values used for boundary conditions for the SANA experiments shown in Table 2 based on the BC options discussed in Section 4.1.

For the fluid and solid energy equations, the central heating rod surface is treated as a known heat flux BC. The heat transfer area from the central rod is $2 \pi R H$, where $R=0.0705 \mathrm{~m}$ and $H=1$ $\mathrm{m}$. The actual powers given in Table 2 are divided by this heat transfer area to give the heat fluxes at the heating rod boundary. This total heat flux must then be divided amongst the solid and fluid phases to provide $\tilde{q}_{f}$ and $\tilde{q}_{s}$ in Eqs. (59) and (60). For simplicity, heat flux splitting is based on the infinite porosity of the bed, e.g., $60 \%$ of the total heat goes to the solid pebbles and $40 \%$ of the total heat goes to the fluid phase.

Due to the large amount of insulation on the top and bottom of the bed, these boundaries are also assumed to be known heat flux BCs, and are assumed insulated for the fluid and solid energy equations. For the fluid at the outer periphery, it is assumed that all heat is transferred to the solid wall and pebbles first, and eventually heat is transferred to the ambient ultimate heat sink. However, in our current approach, the outer solid wall is not explicitly modeled, and therefore a fluid-to-wall heat transfer is not needed. Nevertheless, the Achenbach correlation [2] for fluid-towall heat transfer coefficient is implemented in the code for future uses. For the solid, the outer periphery of the bed is treated as a thermal radiation and natural convection heat transfer boundary, where the ambient temperature is given in Table 2. For natural convection heat transfer at this outer wall, a heat transfer coefficient in the range of $5-25 \mathrm{~W} /\left(\mathrm{m}^{2} \cdot \mathrm{K}\right)$ is generally reported [20]. A constant natural convection heat transfer coefficient of $18.4 \mathrm{~W} /\left(\mathrm{m}^{2} \cdot \mathrm{K}\right)$ is recommended in [19] and is also used in our simulations for demonstration purposes. The emissivity of the solid wall is assumed to be 1 . Further improvement will be done to implement natural convection heat transfer coefficient correlations, and to assess the sensitivities of simulations results to the values of $h_{N C}$ and solid wall emissivity.

As discussed in Section 4.1, no boundary conditions are required for the momentum equation. The gas inlet velocity is less than $3.5 \mathrm{E}-5 \mathrm{~m} / \mathrm{s}$, and is neglected such that all boundaries for the pressure equation are treated as zero flux BCs [19]. An initial condition for pressure is given at 1 atmosphere. 
Table 3. Boundary conditions used for the SANA experiment cases described in Table 2.

\begin{tabular}{ccc}
\hline \hline Case & $\tilde{q}_{f}$ at $\operatorname{Rod}\left(\mathrm{W} / \mathrm{m}^{2}\right)$ & $\tilde{q}_{s}$ at $\operatorname{Rod}\left(\mathrm{W} / \mathrm{m}^{2}\right)$ \\
\hline $\mathrm{A}$ & 12069 & 8056 \\
$\mathrm{~B}$ & 24544 & 16362 \\
$\mathrm{C}$ & 37141 & 24760 \\
$\mathrm{D}$ & 12069 & 8056 \\
$\mathrm{E}$ & 24544 & 16362 \\
$\mathrm{~F}$ & 30802 & 20534 \\
\hline
\end{tabular}




\section{Results and Analysis}

This section presents the verification and validation results completed for the SANA benchmark, followed by a brief code-to-code comparison of results.

\subsection{Numerical Verification}

Before attempting validation exercises testing Pronghorn's ability to model physical systems, a thorough and robust verification framework has been developed. This testing framework verifies correct implementation of the governing equations, BCs, and constitutive relationships discussed in this report and for various other physical models available in Pronghorn. This rigorous verification process is described in detail in [4]. Every new kernel, BC, or constitutive relationship is held to the standard of requiring a Method of Manufactured Solutions (MMS) or physically-intuitive test demonstrating correctness before incorporation into the Pronghorn source code.

The MMS verification technique is commonly used to verify accurate code implementation. MMS allows the user to "choose" the solution and then modify the governing system such that, if the source code is performing as expected, the code returns the solution specified by the user. For instance, consider the diffusion kernel, which in 1-D solves the following equation:

$$
-\frac{d^{2} u}{d x^{2}}=0
$$

Suppose that the user would like to test whether the kernel can return a solution of $\sin (4 \pi x)$. But, only by adding a forcing function would the solution be $\sin (4 \pi x)$. This required forcing function is $f=16 \pi^{2} \sin (4 \pi x)$. So, the equation to solve in the input file is shown by the last equation in Eq. (64). If a solution of $u=\sin (4 \pi x)$ is obtained, then the diffusion kernel is behaving as expected, and it can be inferred that the source code is correct.

$$
\begin{aligned}
-\frac{d^{2}}{d x^{2}}(\sin (4 \pi x)) & \neq 0 \\
-\frac{d^{2}}{d x^{2}}(\sin (4 \pi x))-f & =0 \\
-\frac{d^{2} u}{d x^{2}}-16 \pi^{2} \sin (4 \pi x) & =0
\end{aligned}
$$

For all kernels, BCs, and simple constitutive relationships (such that an analytical expression for the forcing function can be written in an input file), a 2-D and 3-D MMS test is created. For more complicated physics, such as $\kappa$ correlations that tend to be complex, results for a representative problem state are compared in terms of trends and magnitudes with values reported in the literature. For the MMS tests, both the graphical depiction of the solution, and the convergence rates for different Finite Element (FE) shape function orders, are used to assess accuracy. The error 
between the FE solution and the exact solution can be expressed in terms of the element size $h_{e}$ and a derivative of the exact solution:

$$
\text { error } \leq C h_{e}^{i+1}\left\|u^{(i)}\right\|
$$

where $i$ is the order of the shape functions ( 1 for linear, 2 for quadratic, etc.), $u^{(i)}$ is the $i$-th derivative of the exact solution $u$, and $C$ is an unknown constant. When the logarithm of the error is plotted against the logarithm of the element size, slopes of 2 and 3 should be obtained for linear and quadratic elements, theoretically. Over 120 MMS tests exist for the Pronghorn source code, and a complete summary of these tests is beyond the scope of this report. Fig. 3 and 4 show MMS convergence rates for the $\left(-\frac{\varepsilon}{W}\left[-\varepsilon \nabla P+\varepsilon \rho_{f} \vec{g}\right], \nabla \psi\right)$ pressure Poisson kernel in 2-D and the $\left(-\kappa \nabla T_{S}, \nabla \psi\right)$ solid energy diffusion kernel in 2-D $r-z$ geometry, respectively. For both cases, multiple MMS tests are shown to ensure further accuracy. So, while there are over 120 MMS tests, there are closer to 300 actual verifications of correct implementation because each MMS test contains several independent tests.

Figure 3. Error as a function of mesh spacing $\Delta x$ for $\varepsilon=0.35, W=2.0$, and (a) $P=1000 x^{2}+50 e^{y}$ and $T_{f}=1000$, (b) $P=1000 x^{3}+50 e^{y}$ and $T_{f}=50 e^{x}+1000 y^{3}+2000$, and (c) $P=100 e^{x}+200 y+$ 500 and $T_{f}=1000+50 e^{y}$ for the pressure Poisson kernel [4]. 
Figure 4. Error as a function of mesh spacing $\Delta x$ for (a) $T_{f}=\sin (2 \pi x) \sin (2 \pi y)$ and $\kappa=100 x=$ $200 y+5000$, (b) $T_{f}=e^{x} e^{y}$ and $\kappa=200 x^{3}+300 y^{4}$, and (c) $T_{f}=x^{3}+e^{y}$ and $\kappa=\sin (x)+\cos (y)$ for the solid energy diffusion kernel in 2-D $r-z$ geometry [4].

In addition to the MMS tests for spatial kernels and BCs, accuracy of the time-dependent kernels, such as those representing time derivatives, is assessed by selecting a MMS solution that has very close to zero spatial error and then evaluating the error for different time integration schemes as a function of the time step size. If the true solution is linear, then using linear spatial shape functions will give very close to zero spatial error such that only the time error is measured. For example, the convergence rates for the $\left(\varepsilon \rho_{f} C_{p, f} \frac{\partial T_{f}}{\partial t}, \psi\right)$ kernel for different time integration schemes is shown as a function of the time step $\Delta t$ in Fig. 5. 
Figure 5. Convergence rates for different time integration schemes for $T_{f}=(x+y+15) e^{-1}$, $\rho_{f}=100 x+200 y+1, \varepsilon=0.35 x+0.25 y+0.1$, and $C_{p}=e^{x}+e^{y}+100$ for the $\left(\varepsilon \rho_{f} C_{p, f} \frac{\partial T_{f}}{\partial t}, \psi\right)$ kernel [4]. This is the time derivative kernel in the fluid energy equation.

Having thoroughly demonstrated correct numerical implementation of all physics in Pronghorn [4], the next sections present the validation results of the SANA simulations.

\subsection{Validation}

Numerical simulations have been performed for all experiment cases listed in Table 2. All simulation results are obtained at the steady state via accelerated transient simulations, including that the transient term in the solid energy equation is not included, and that nitrogen cases use larger thermal conductivity at the beginning of simulations to quickly establish a natural ciruclation and then is reduced to its normal value. Figs. 6-8 show Pronghorn solid temperature simulation results for cases A, B, and C, respectively. Also shown are experiment measurements of pebble temperatures at three vertical locations. Helium simulation results were obtained via a transient simulation to enhance stability using a $40 \times 20(r-z)$ uniform mesh shown in Fig. 12(a). A mesh independence study described in Appendix B demonstrates the appropriateness of this mesh.

For the helium results shown in Figs. 6-8, temperatures tends to be overpredicted in the center of the bed and slightly underpredicted towards the periphery of the bed. The simulation results were found to be sensitive to the correlation chosen for $\kappa$. In the center of the bed where temperatures are higher, slight variations in $\kappa_{\text {radiation }}$ can yield substantially different temperatures. More accurate correlations for $\kappa$ that differentiate between radiation heat transfer between pebbles versus radiation heat transfer between pebbles and a nearby wall, will be investigated. Larger errors towards the edges of a packed bed are also to be expected simply due to the nature of the porous media assumption of treating a packed bed as a continuum. The largest errors occur for the $10 \mathrm{~kW}$ 
power, likely because at low powers natural convection heat transfer constitutes a greater fraction of the total heat transfer.

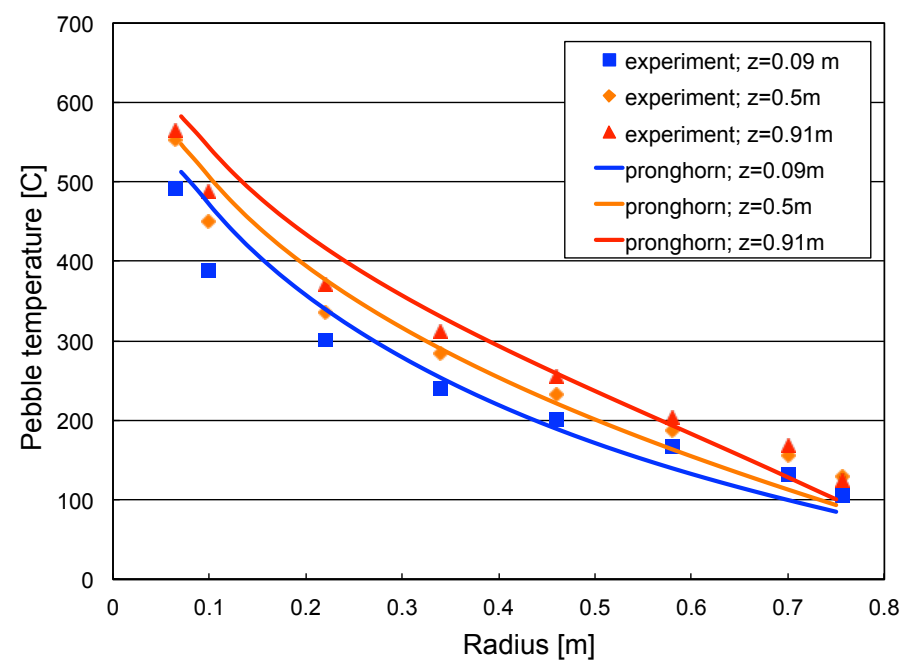

Figure 6. Pronghorn solid temperature computed for helium with $10 \mathrm{~kW}$ nominal heater rod power (case A).

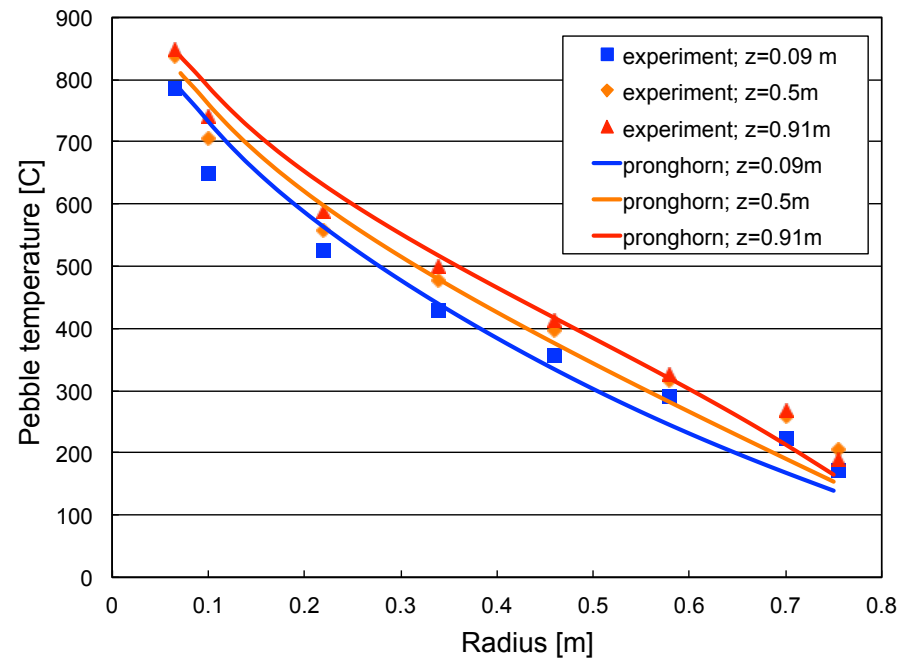

Figure 7. Pronghorn solid temperature computed for helium with $20 \mathrm{~kW}$ nominal heater rod power (case B). 


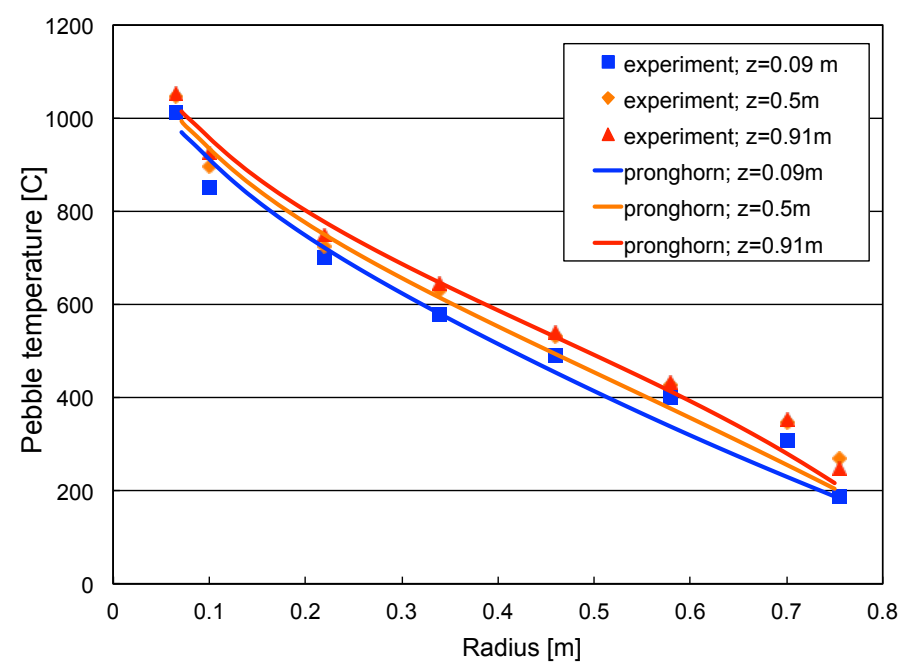

Figure 8. Pronghorn solid temperature computed for helium with $30 \mathrm{~kW}$ nominal heater rod power (case C).

Nitrogen simulation results for cases D, E, and F are shown in Figs. 9-11. A different mesh was used for these cases, since the Peclet number for nitrogen is about an order of magnitude larger than that for helium. A large Peclet number signifies a convection-dominated flow, which is well-known to cause numerical difficulties when solved with an unstabilized FEM. The mesh used for the nitrogen cases is shown in Fig. 12(b). In addition, the Zehner-Bauer-Schlünder correlation showed unstable and slow convergence rate when applied to nitrogen cases. Instead, a curve-fitting correlation for effective thermal conductivity against pebble temperature based on THERMIX [13] data is used. The reason for unstable and slow convergence of the Zehner-Bauer-Schlünder correlation is currently under investigation.

Pronghorn solid temperatures for the nitrogen cases are shown in Fig.s 9-11. When compared to helium cases, the agreement between the numerical and experimental results is poorer, which is likely primarily due to the use of constant nitrogen viscosity and thermal conductivity. We anticipate the numerical results will be improved when the more accurate nitrogen gas thermal properties package becomes available.

The gradients in temperatures moving in the axial direction is much larger than that seen for the helium cases, demonstrating the increased importance of natural convection heat transfer for nitrogen. The largest discrepancies for the nitrogen simulations occur in the center of the bed. Because this is not a high-radiation environment (due to the lower temperatures than towards the bed center) and because the porous media approximation should be very accurate in the bed center, these errors corroborate our speculation of improved simulation results upon delivery of a sophisticated nitrogen thermal properties library. 


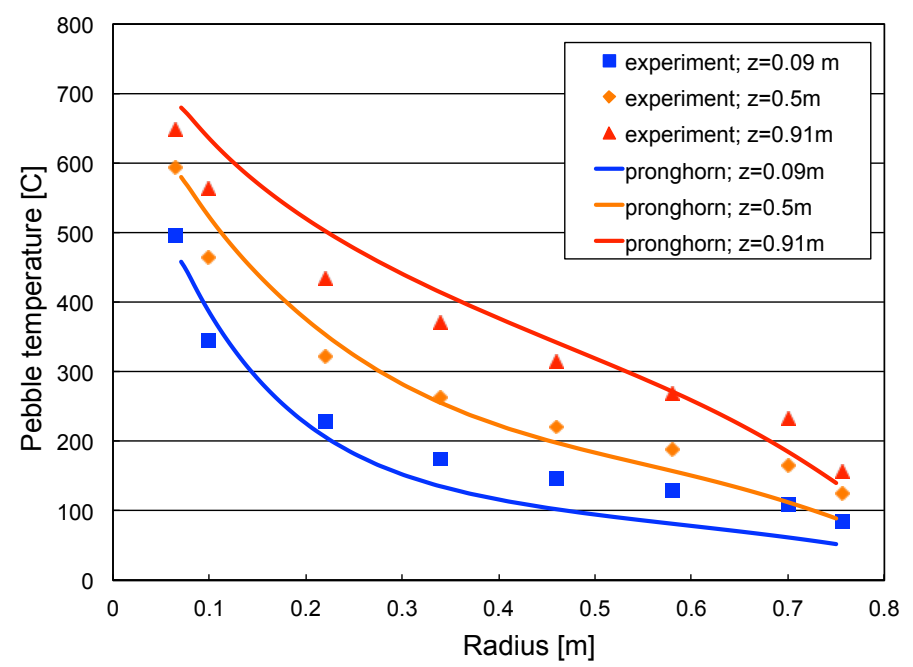

Figure 9. Pronghorn solid temperature computed for nitrogen with $10 \mathrm{~kW}$ nominal heater rod power (case D).

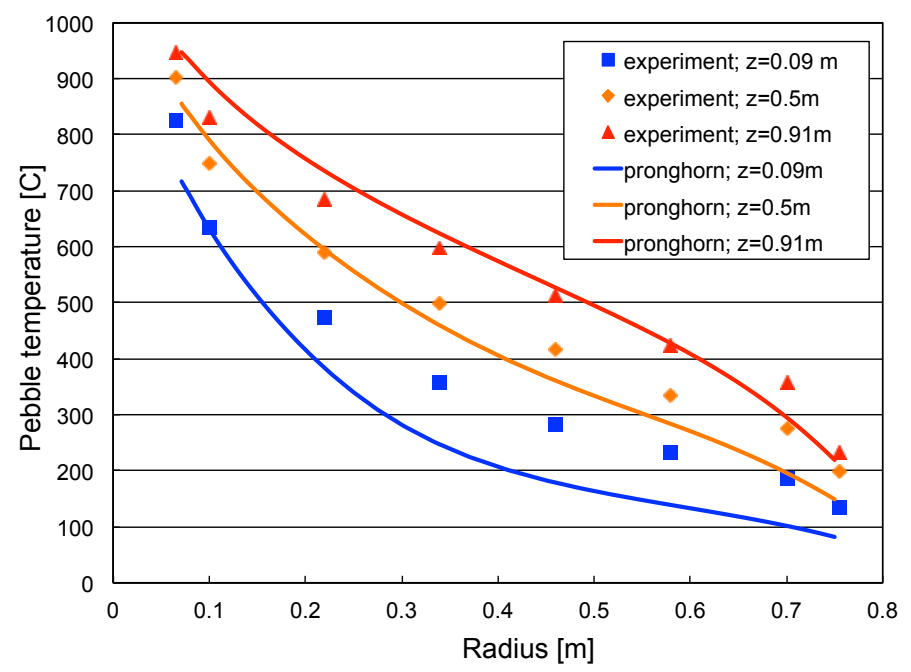

Figure 10. Pronghorn solid temperature computed for nitrogen with $20 \mathrm{~kW}$ nominal heater rod power (case E). 


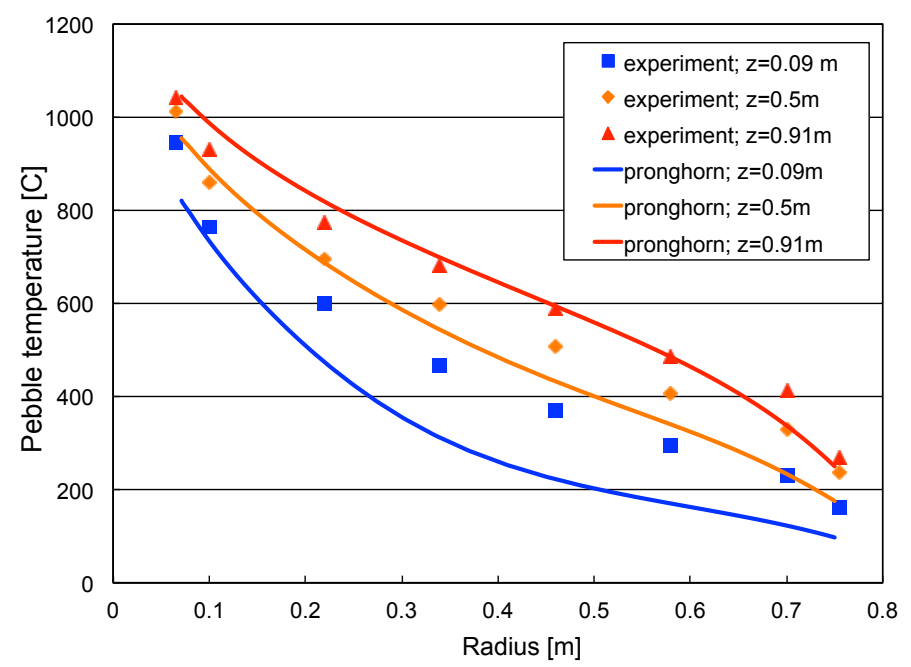

Figure 11. Pronghorn solid temperature computed for nitrogen with $25 \mathrm{~kW}$ nominal heater rod power (case F).

Overall, the simulation results agree with the experiment measurements reasonably well. Pronghorn is capable of predicting the overall trends of temperature distribution in the radial direction. The outer wall thermal radiation and natural circulation heat transfer BCs work reasonably well in capturing the outer wall temperatures.

Fig. 13 shows Pronghorn simulation results for the velocity field for case A. A natural circulation of coolant is established due to heating on the inner wall and cooling on the outer wall. Experimental measurements on coolant velocities were not available, and therefore validation on these quantities cannot be provided. These natural circulation flow rates are sufficiently low that natural circulation can be neglected for helium; this has been confirmed by other researchers [19]. 


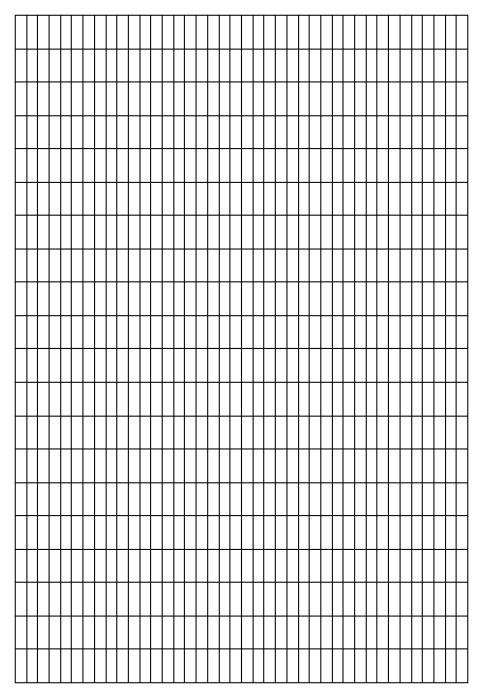

(a)

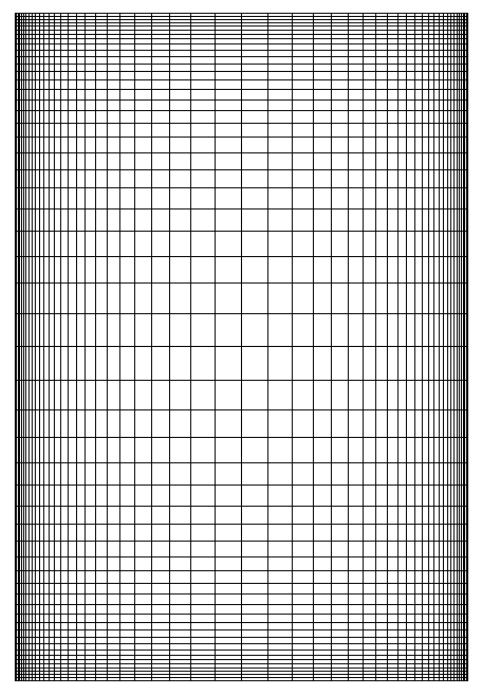

(b)

Figure 12. Meshes used for SANA experiment simulations for (a) the helium cases, and (b) the nitrogen cases. 


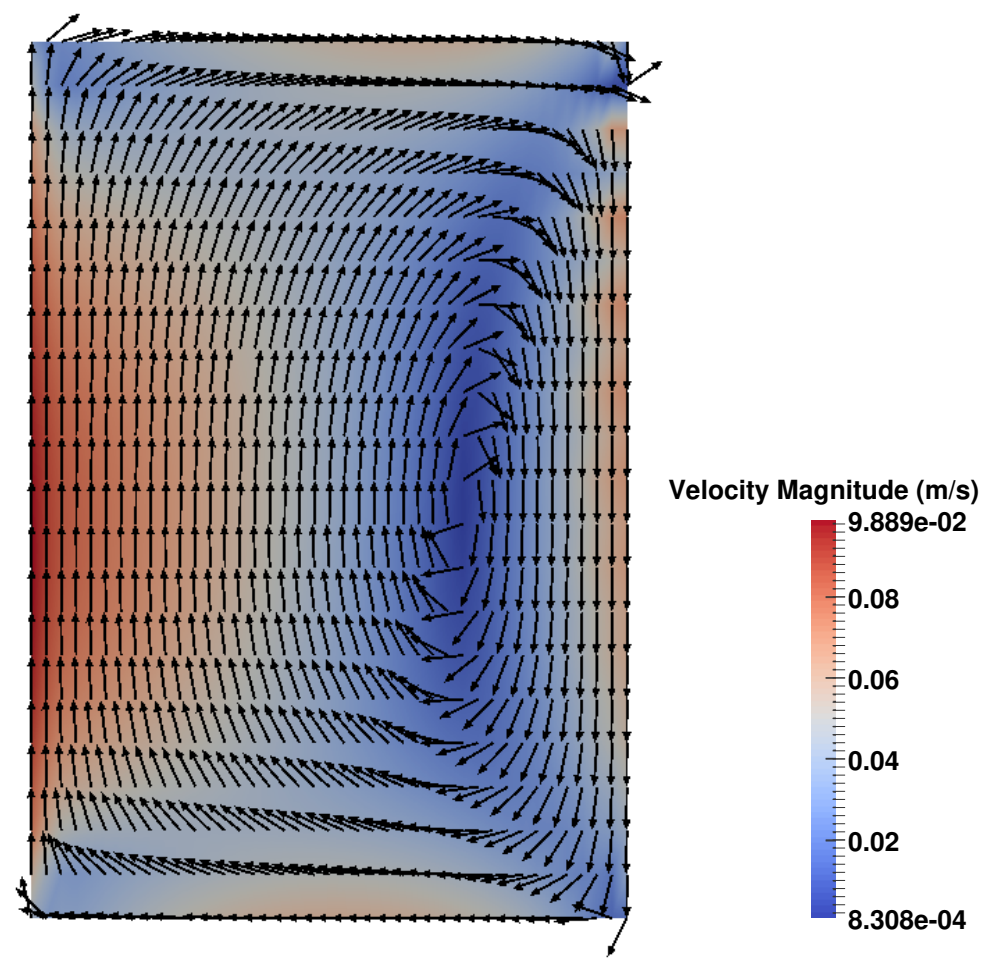

Figure 13. Pronghorn results for the velocity field of case A (10kW nominal heating power and helium coolant). The plot is colored with velocity magnitude in $(\mathrm{m} / \mathrm{s})$.

\subsection{Code-to-Code Comparisons}

This section presents a brief discussion of code-to-code comparisons of Pronghorn with the results of other researchers that have completed the SANA benchmark. Results are compared with a legacy version of Pronghorn (circa 2009), MGT-3D, ANSYS CFX, THERMIX, TRIO-EF, and TINTE $[13,19,21]$. All of these listed codes use a porous media thermal hydraulics model and 2-D $r-z$ geometry to solve a subset of the SANA test cases. Various levels of geometric detail and modeling assumptions are used. Unless otherwise noted, the governing equations used in these codes are reasonably similar to the equations shown in Eqs. (3), (5), (6), and (7), but significant differences are noted when applicable.

This section does not aim to summarize results of all SANA benchmark simulations available in the literature, but only to cast the present results in terms of several other comparable porous media tools, while providing prospective reasoning for the discrepancies with experimental data shown in Figs. 6, 7, and 8. Additional benchmark results for Flownex and AGREE are referenced in case of interest $[6,22]$. Because only graphical data is available for these other codes, a detailed numerical comparison cannot be performed; hence, the discussion in this section is mostly qualitative. To 
keep this section brief, only results for the $10 \mathrm{~kW}$ helium test case are compared amongst these codes, though results are only available in the literature for the $30 \mathrm{~kW}$ helium case for MGT-3D and CFX [19].

The legacy version of Pronghorn (circa 2009) completed test cases A and C shown in Table 2 [21]. Only the pebble bed core is modeled explicitly, and the effects of surrounding materials are approximated via the BCs. Comparing the legacy Pronghorn results for case A in Fig. 14 with the present results in Fig. 6 shows that the temperature results are comparable, while the legacy results are actually slightly closer to the experimental values near the center of the bed. However, the legacy version of Pronghorn used different BCs than those discussed in Section 4.1; Dirichlet conditions were set on the fluid and solid temperatures according to the data in the SANA report. When modeling a real system, the fluid and solid temperatures are not known a-priori, so this legacy choice of BCs does not accurately assess the code's capability of modeling the SANA facility. The difference in results arising from the $\mathrm{BC}$ selection suggests that the present results could be improved with a more sophisticated BC at the heater element surface.

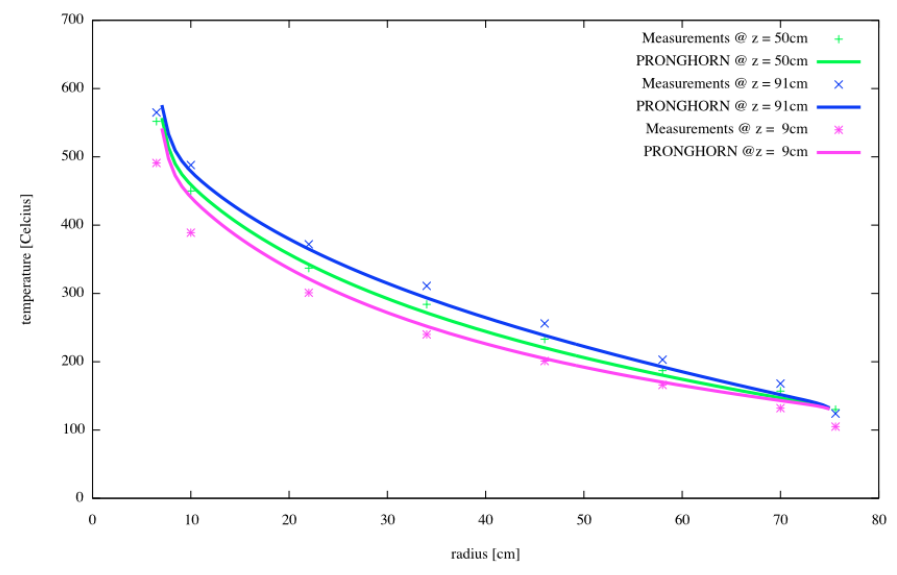

Figure 14. Legacy Pronghorn temperature results for case A [21].

MGT-3D, a modern evolution of TINTE, and ANSYS CFX, a Computational Fluid Dynamics (CFD) code run using a porous media model, have recently been used to model the SANA facility [19]. Both of these codes included a much more detailed geometrical description of the experiments, and explicitly modeled the heating elements, top and bottom connection electrodes, and insulation layers. Due to the different geometry, different BCs were also used. The heat flux at the heater element surface is computed by assuming radial radiation heat transfer from the resistance heater across the gap to provide a more accurate BC. Rather than using a constant porosity, the porosity in the center of the bed is set to 0.39 , and within a half pebble diameter of the walls, is set to 0.88 (near the heater element) or 0.48 (near the outer wall). The Zehner-Schlünder correlation is used for $\kappa$, but an additional correction radiation component is added for regions within one half pebble diameter of the wall.

The CFX model uses the Unsteady Reynolds-Averaged Navier Stokes (URANS) porous media equations with the Shear Stress Transport (SST) turbulence model. Contrary to the process 
followed in this report, the wall heat transfer coefficient and emissivity of the heating element for both the MGT-3D and CFX results were fine-tuned such that the numerical results matched the experimental results as best as possible for test case $\mathrm{C}$. These values were then held constant for the remaining tests. Similar to the discussion of the legacy Pronghorn results, this choice of BCs does not fully validate the MGT-3D and CFX codes, since real simulations will not always have exact results to provide fine-tuning. Comparing the MGT-3D and CFX results for helium at $30 \mathrm{~kW}$ in Fig. 15 with the present results in Fig. 8 show comparable temperatures. CFX results show the best prediction of experimental results. This may be attributed to the lack of a sophisticated turbulence model in Pronghorn or MGT-3D.
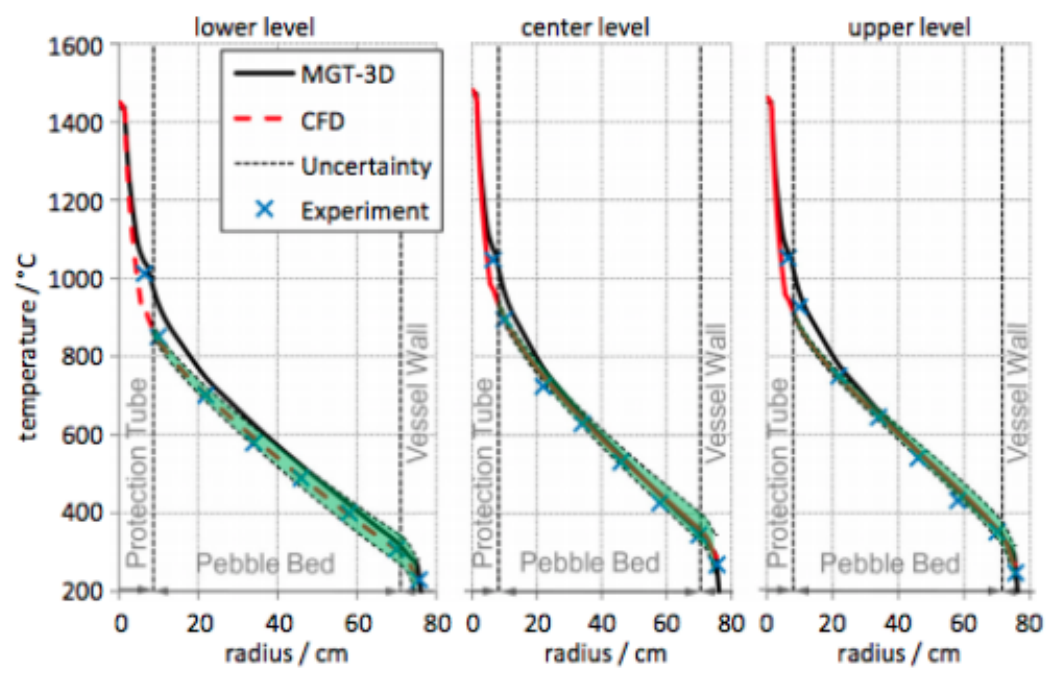

Figure 15. MGT-3D and ANSYS CFX (CFD) temperature results for case C [19]. The uncertainty band was estimated by varying the BCs within the bounds recommended in the SANA documents.

Several of the steady state SANA cases have also been used to validate the THERMIX code [13]. Similar to the MGT-3D and CFX models discussed above, the core, heating elements, insulation, and other more minor components are all explicitly modeled. A porosity of 0.41 is used in the center of the bed, with 0.65 near the heating element and 0.5 at the outer edge. Comparing the THERMIX results for case A shown in Fig. 16 with the present results in Fig. 6 shows that the temperatures are of comparable accuracy. 


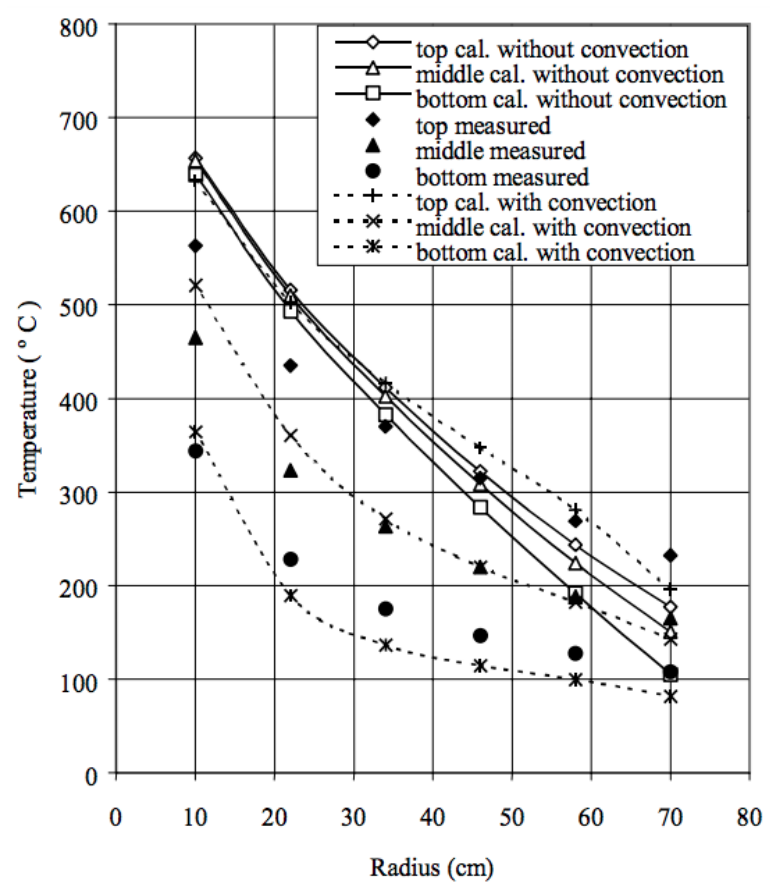

Figure 16. THERMIX temperature results for case A [13].

TRIO-EF, a finite element code developed in France, simulated a variety of the SANA test cases. The governing equations in TRIO-EF only includes one energy equation, such that the two phases are assumed to be at the same temperature. In addition, the Brinkman model for porous drag is used, such that viscous effects are directly modeled in the fluid. Both of these modeling assumptions lead to different BCs - most importantly, BCs are not separated for the fluid and solid. Porosity is expressed by an exponential function of the distance from the wall. A similar correlation is used for computing $\kappa$, with a correction applied within one half pebble diameter of the walls. Contrary to the MGT-3D and CFX simulations, however, $\kappa$ is halved near the walls, instead of adding a correction. By comparing TRIO-EF results for case A shown in Fig. 17 with the present results in Fig. 6, it can be seen that again, temperatures agree fairly well. 


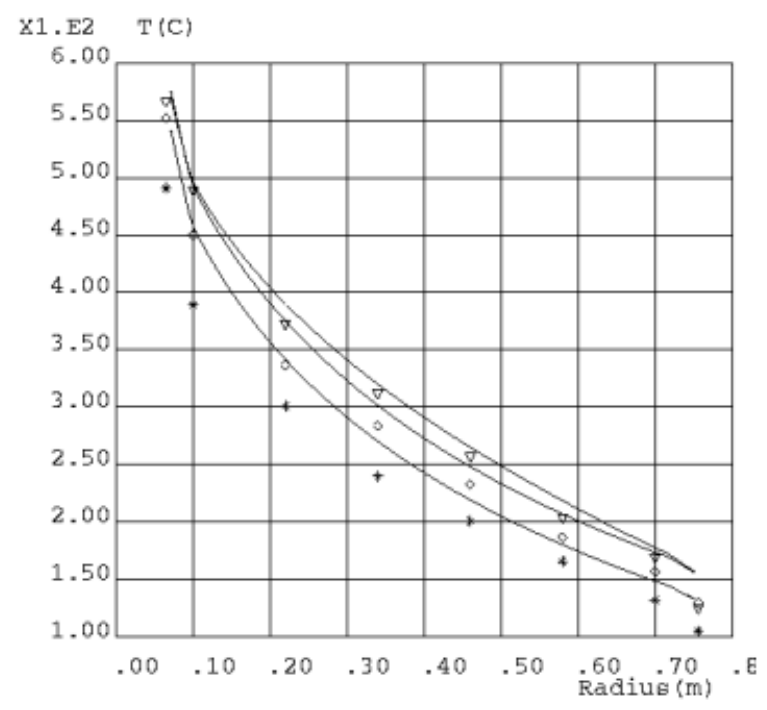

Figure 17. TRIO-EF temperature results for case A [13].

Finally, TINTE modeled the SANA facility with a level of geometric detail similar to the previously discussed MGT-3D results. Eq. (15) is used for $\kappa$, with an additional correction made within one half pebble diameter of the bed. Porosity is assumed constant at 0.41 in the center of the bed, and within one half pebble diameter of the wall, is 0.65 near the heating element and 0.5 near the outer wall. By comparing TINTE results for case A in Fig. 18 with the present results in Fig. 6, shows similar temperatures.

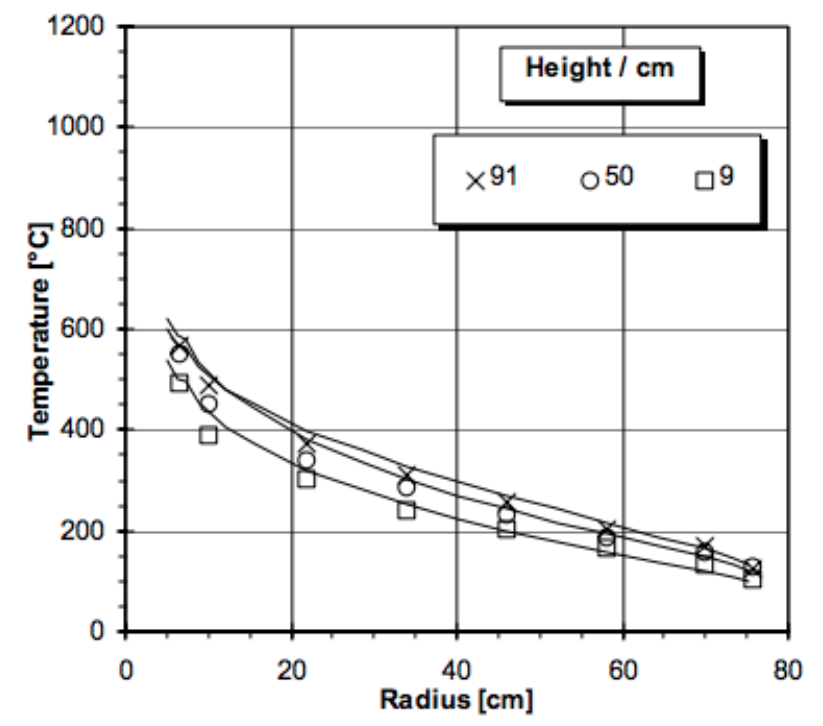

Figure 18. TINTE temperature results for case A [13]. 
The brief review of SANA simulation results obtained with different comparable porous media codes shows that the present results are of comparable accuracy. All of these codes and the present work overpredict temperatures in the center of the bed, with much better agreement at the outer edges of the bed. In summary, the largest differences between the approach taken in the present work and the previously-discussed results are:

1. Geometric fidelity - all results discussed here except for the present and legacy Pronghorn results explicitly model the insulation, heating element internals, electrodes, and other aspects of the geometry that were here approximated as negligible.

2. Choice of BCs - the legacy Pronghorn results inappropriately use the benchmark specifications to specify exact BCs that should otherwise not be known to a code user. Similarly, the MGT-3D and CFX results were obtained by fine-tuning the wall heat transfer coefficient and the radiative $\mathrm{BC}$ at the heater element surface to match simulation results. In realistic code usage, experimental results will not always be available. For this reason many neutronics benchmarks will not provide the users with the simulation results in order to eliminate user model manipulation.

3. Choice of porosity - all of the codes discussed in this section except the two versions of Pronghorn use either a piecewise constant porosity or an exponentially-varying porosity. A sensitivity analysis performed using TRIO-EF suggests that using an exponential versus a piecewise constant variation for porosity does not have a significant impact on the temperatures, and hence it is assumed in this work that a piecewise constant porosity also does not produce significantly different results than assuming a constant porosity [13]. Further investigations will be performed in future work, and a number of exponential and oscillatory exponential correlations for porosity have already been incorporated into Pronghorn.

4. Choice of $\kappa$ - while all codes used comparable correlations for the various components of $\kappa$, MGT-3D, CFX, TRIO-EF, and TINTE apply additional corrections within a half pebble diameter of the wall.

5. Turbulence models - only the CFX results use accurate turbulence modeling.

The discrepancies near the center of the bed suggest additional corrections should be made

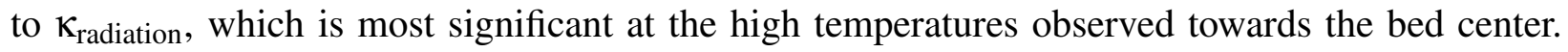
In addition, a more accurate radiation heat transfer $\mathrm{BC}$ at the heater element surface is needed to provide a more precise estimate of the fraction of the heat flux that enters the solid and fluid phases. Currently, the infinite porosity is used to approximate this division, but more complicated physics are involved. In addition, the present results could likely be improved by used a more sophisticated turbulence model, rather than relying on heat transfer coefficient and drag correlations to capture this complex effect.

Within the limits of not utilizing the benchmark specifications to apply unrealistically-knowable $\mathrm{BCs}$, the present Pronghorn results demonstrate that Pronghorn is capable of simulating heliumcooled HTRs at decay heat levels. Future validation exercises will extend Pronghorn's validation space. 


\section{Summary}

In this work, we have re-established Pronghorn's capability to simulate heat and mass transfer in pebble beds based on the porous medium approximation. Although more sophisticated physics models are available, we have limited ourselves to using a simpler Darcy-Forchheimer flow model inherited from older versions of the code. Future work will involve the use of the porous medium Euler equations that do not assume negligible momentum advection and time rate of change.

Closure correlations, such as friction coefficients in pebble beds and fluid-to-pebble heat transfer coefficients, are reviewed, assessed, and implemented in Pronghorn to support simulations of interest to nuclear reactor thermal hydraulics analysis. Numerical verification was performed in form of mesh convergence study using MMS for both spatial and temporal integration schemes, and the expected orders of convergence were observed. Validation of this code was performed by comparing simulation results against experimental measurements of the SANA experiment. For the helium cases at three power levels, simulation results of solid temperatures agree well with experiment measurements and other simulation tools that have performed the same benchmark. For the nitrogen cases, because less accurate thermal properties were used, relatively large discrepancies between the simulation results and experiment measurements were found. However, we anticipate that this will be improved when the more accurate nitrogen gas thermal properties package becomes available. This benchmark validation exercise has also initiated concentrated efforts on FE stabilization schemes to help improve numerical converge such that simulations converge faster. Many such stabilization methods are available in the literature, and are currently under consideration in Pronghorn [23-26]. 


\section{A Notation}

This section presents a concise reference of all notation used in this document. An arrow superscript over a symbol indicates a vector.

\section{A.1 Greek Symbols}

$\begin{array}{lll}\alpha & \text { bed convective heat transfer coefficient } & \mathrm{W} /\left(\mathrm{m}^{3} \mathrm{~K}\right) \\ \alpha_{c} & \text { correlation term } & 1 \\ \vec{\delta} & \text { linearized update vector } & \overrightarrow{1} \\ \varepsilon & \text { porosity } & 1 \\ \varepsilon & \text { emissivity or JFNK parameter, context-specific } & 1 \\ \varepsilon_{\text {mach }} & \text { machine precision } & 1 \\ \kappa & \text { effective solid thermal conductivity } & \mathrm{W} /(\mathrm{m} \cdot \mathrm{K}) \\ \phi & \text { finite element shape function } & 1 \\ \varphi & \text { contact area fraction } & 1 \\ \rho & \text { density } & \mathrm{kg} / \mathrm{m}^{3} \\ \sigma & \text { Stefan-Boltzmann constant } & \mathrm{W} /\left(\mathrm{m}^{2} \cdot \mathrm{K}^{4}\right) \\ \mu & \text { dynamic viscosity } & \mathrm{Pa} \cdot \mathrm{s} \\ \Lambda & \text { correlation parameter } & 1 / \mathrm{m}^{4} \\ \lambda & \text { thermal conductivity ratio, } k_{f} / k_{s} & 1 \\ \nu & \text { Poisson ratio } & 1 \\ \vec{\eta} & \text { residual norm vector in GMRES minimization } & \overrightarrow{1} \\ \psi & \text { finite element test function } & 1 \\ \Omega & \text { a volume } & \mathrm{m}^{3} \\ \partial \Omega & \text { a surface } & \mathrm{m}^{2}\end{array}$




\section{A.2 English Symbols}

\begin{tabular}{|c|c|c|}
\hline$a$ & correlation parameter & 1 \\
\hline$a_{w}$ & heat transfer length & $1 / \mathrm{m}$ \\
\hline$A$ & area & $\mathrm{m}^{2}$ \\
\hline $\mathbf{A}$ & linearized matrix & 1 \\
\hline$\vec{b}$ & linearized right-hand-side vector & $\overrightarrow{1}$ \\
\hline$B$ & correlation parameter & 1 \\
\hline$C$ & correlation parameter & 1 \\
\hline$C_{p}$ & isobaric specific heat & $\mathrm{J} /(\mathrm{kg} \cdot \mathrm{K})$ \\
\hline$d$ & pebble diameter & $\mathrm{m}$ \\
\hline$D$ & bed diameter & $\mathrm{m}$ \\
\hline $\mathscr{D}$ & Krylov suspace & - \\
\hline$E$ & Young's modulus & $\mathrm{Pa}$ \\
\hline$F$ & pebble bed weight & $\mathrm{kg} \cdot \mathrm{m} / \mathrm{s}^{2}$ \\
\hline$\vec{F}$ & residual vector & $\overrightarrow{1}$ \\
\hline$\vec{g}$ & gravitational acceleration & $\mathrm{m} / \mathrm{s} 2$ \\
\hline$h$ & bed convective heat transfer coefficient & $\mathrm{W} /\left(\mathrm{m}^{2} \mathrm{~K}\right)$ \\
\hline $\mathbf{H}$ & upper-Hessenberg matrix & 1 \\
\hline$\tilde{\mathbf{H}}$ & non-square upper-Hessenberg matrix & 1 \\
\hline $\mathbf{J}$ & Jacobian matrix & 1 \\
\hline$k$ & thermal conductivity & $\mathrm{W} /(\mathrm{m} \cdot \mathrm{K})$ \\
\hline $\mathscr{K}_{D}$ & modified gas thermal conductivity & 1 \\
\hline $\mathscr{K}_{R}$ & normalized radiation thermal conductivity & 1 \\
\hline $\mathscr{K}_{S D}$ & correlation parameter & 1 \\
\hline$m$ & correlation parameter & 1 \\
\hline$M$ & molar mass & $\mathrm{g} / \mathrm{mol}$ \\
\hline$n$ & finite element node number & 1 \\
\hline$\vec{n}$ & unit normal vector & 1 \\
\hline$N$ & total number of finite element nodes & 1 \\
\hline$N_{c}$ & coordination number & 1 \\
\hline$N_{A}$ & number of spheres per unit area & $1 / \mathrm{m}^{2}$ \\
\hline$N_{L}$ & number of spheres per unit length & $1 / \mathrm{m}$ \\
\hline$N u$ & Nusselt number, $N u=h d / k_{f}$ & 1 \\
\hline$N u_{\text {wall }}$ & wall Nusselt number, $N u=h_{\text {wall }} d / k_{f}$ & 1 \\
\hline $\mathscr{O}$ & "on the order of" & - \\
\hline$P$ & pressure & $\mathrm{Pa}$ \\
\hline $\operatorname{Pr}$ & fluid Prandtl number, $\operatorname{Pr}=\mu_{f} C_{p, f} / k_{f}$ & 1 \\
\hline$q$ & heat flux & $\mathrm{W} / \mathrm{m}^{2}$ \\
\hline$\vec{q}$ & column vector in $\mathbf{Q}$ & $\overrightarrow{1}$ \\
\hline
\end{tabular}




$\begin{array}{lll}\mathbf{Q} & \text { orthonormal basis } & - \\ r & \text { radial coordinate } & \mathrm{m} \\ \vec{r} & \text { residual vector } & \overrightarrow{1} \\ r_{c} & \text { contact radius } & \mathrm{m} \\ r_{\text {pebble }} & \text { pebble radius } & \mathrm{m} \\ R & \text { gas constant } & \mathrm{J} / \mathrm{mol} \cdot \mathrm{K} \\ \mathbb{R}^{n} & \text { the space of real numbers of dimension } n & - \\ R e & \text { Reynolds number, } R e=\rho_{f} \varepsilon \vec{V} d / \mu_{f} & 1 \\ \vec{s} & \text { starting vector for Arnoldi process } & \overrightarrow{1} \\ S_{F} & \text { vertical force component } & 1 \\ T & \text { intrinsic temperature } & \mathrm{K} \\ u_{j} & \text { expansion coefficients (components of the solution vector) } & - \\ \vec{u} & \text { solution vector } & - \\ \vec{V} & \text { intrinsic velocity } & \mathrm{m} / \mathrm{s} \\ W & \text { porous media friction coefficient } & 1 / \mathrm{s} \\ x & x \text {-coordinate } & \mathrm{m} \\ \vec{x} & \text { linearized solution vector } & \overrightarrow{1} \\ y & y \text {-coordinate } & \mathrm{m} \\ \vec{y} & \text { linearized update vector } & \overrightarrow{1} \\ z & \text { axial coordinate } & \mathrm{m}\end{array}$




\section{A.3 Subscripts and Other Notation}

This section lists the subscripts used in this document. Subscripts whose interpretation is obvious, such as "convection," are not repeated here.

$\begin{array}{ll}c & \text { cross-sectional } \\ f & \text { fluid } \\ \text { fluid conduction } & \text { pebble-to-fluid-to-pebble conduction } \\ i & \text { inlet } \\ \text { in } & \text { inner or inflow, context-specific } \\ o & \text { outlet } \\ \text { out } & \text { outer or outflow, context-specific } \\ s & \text { solid } \\ \text { solid conduction } & \text { pebble-to-pebble conduction } \\ r & \text { radial component } \\ \text { radiation } & \text { thermal radiation } \\ \text { wall } & \text { wall } \\ x & x \text {-component } \\ y & y \text {-component } \\ z & \text { axial component } \\ \infty & \text { infinite-medium value } \\ \|(.)\|_{2} & \text { L-2 norm of }(.)\end{array}$




\section{B Mesh Independence Study}

Using case A for demonstration purpose, mesh independence study was performed using several sets of meshes, including $10 \times 5(r-z), 20 \times 10,40 \times 20$, and $80 \times 40$. At the steady state, numerical results of the solid temperature are sampled in the vertical center of the domain $(\mathrm{z}=$ 0.5). These results are shown in figure B.1, from which it is concluded that the $40 \times 20$ mesh is an appropriate choice.

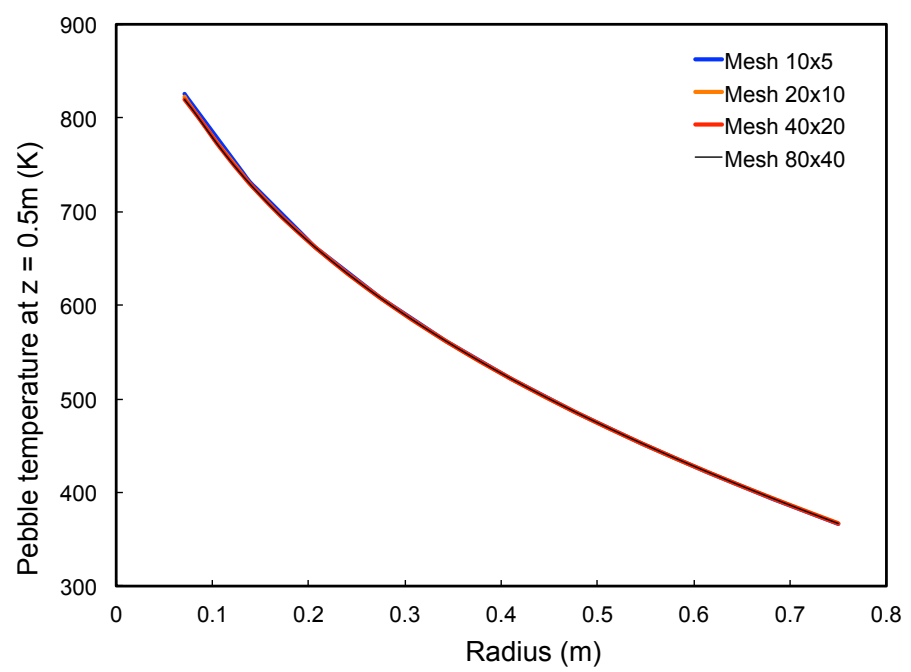

Figure B.1. Mesh independence study for case A. 


\section{References}

[1] B. Stocker and H. Nieben. Data Sets of the SANA Experiment 1994-1996. Technical report, Forschungszentrum Julich, 1996.

[2] E. Achenbach. Heat and flow characteristics of packed beds. Experimental Thermal and Fluid Sciences, 10:17-27, 1995.

[3] P. Avigni. TRACE Loop Modeling of the Liquid Salt Test Loop. Technical report, Oak Ridge National Laboratory, 2016.

[4] A.J. Novak, L. Zou, J.W. Peterson, D. Andrs, C. Permann, and R.C. Martineau. Pronghorn Theory Manual. Technical report, Idaho National Laboratory, 2017.

[5] A. Klerk. Voidage Variation in Packed Beds at Small Column to Particle Diameter Ratio. American Institute of Chemical Engineers, 49:2022-2029, 2003.

[6] V. Seker and T. Downar. Multiphysics Methods Development for High Temperature Gas Cooled Reactor Analysis. In PROCEEDINGS in the International Conference on Emerging Nuclear Energy Systems, 2007.

[7] KTA. Reactor Core Design of High-Temperature Gas-Cooled Reactors Part 3: Loss of Pressure through Friction in Pebble Bed Cores. Technical Report KTA 3102.3, Nuclear Safety Standards Commission, 1981.

[8] KTA. Reactor Core Design of High-Temperature Gas-Cooled Reactors Part 3: Heat Transfer in Spherical Fuel Elements. Technical Report KTA 3102.2, Nuclear Safety Standards Commission, 1983.

[9] E. You, X. Sun, F. Chen, L. Shi, and Z. Zhang. An improved prediction model for the effective thermal conductivity of compact pebble bed reactors. Nuclear Engineering and Design, 323:95-102, 2017.

[10] C. T. Hsu, P. Cheng, and K. W. Wong. Modified Zehner-Schlunder Models for Stagnant Thermal Conductivity of Porous Media. International Journal of Heat and Mass Transfer, 37:2751-2759, 1994.

[11] G. Breitbach and H. Barthels. Radiant heat transfer in the HTR core after failure of the afterheat removal systems. Transactions of the American Nuclear Society, 31:421-422, 1979.

[12] C. K. Chan and C. L. Tien. Conductance of Packed Spheres in Vacuum. Transactions of the ASME, pages 302-308, 1973.

[13] Nuclear Power Technology Development Section. Heat Transport and Afterheat Removal for Gas Cooled Reactors Under Accident Conditions. Technical Report IAEA-TECDOC-1163, IAEA, 2000. 
[14] H. Petersen. The Properties of Helium: Density, Specific Heats, Viscosity, and Thermal Conductivity at Pressures from 1 to 100 bar and from Room Temperature to about $1800 \mathrm{~K}$. Technical report, Danish Atomic Energy Commission, 1970.

[15] KTA. Reactor Core Design of High-Temperature Gas-Cooled Reactors Part 1: Calculation of the Material Properties of Helium. Technical Report KTA 3102.1, Nuclear Safety Standards Commission, 1978.

[16] National Institute of Standards and Technology. Thermophysical properties of fluid systems, 2017. Available at http://webbook.nist.gov/chemistry/fluid/.

[17] A. T. D. Butland and R. J. Maddison. The Specific Heat of Graphite: An Evaluation of Measurements. Journal of Nuclear Materials, 49:45-56, 1973.

[18] Y. Saad and M. Schultz. GMRES: A Generalized Minimum Residual Algorithm for Solving Nonsymmetric Linear Systems. Journal of Scientific and Statistical Computing, 7:856-869, 1986.

[19] J. Baggemann, D. Shi, S. Kasselmann, S. Kelm, H.J. Allelein, and A. Hurtado. Use of SANA Experimental Data for Validation and Verification of MGT-3D and a CFD Porous Media Model for VHTR Application. Nuclear Engineering and Design, pages 678-687, 2016.

[20] Thomas Ask. Engineering for Industrial Designers and Inventors: Fundamentals for Designers of Wonderful Things. O'Reilly, 2016.

[21] H. Park, D. A. Knoll, D. R. Gaston, and R. C. Martineau. Tightly coupled multiphysics algorithms for pebble bed reactors. Nuclear Science and Engineering, 166:118-133, 2010.

[22] C.G. du Toit, P.G. Rousseau, G.P. Greyvenstein, and W.A. Landman. A Systems CFD Model of a Packed Bed High Temperature Gas-Cooled Nuclear Reactor. International Journal of Thermal Sciences, 45:70-85, 2006.

[23] V. Dalen. Simplified finite-element models for reservoir flow problems. Society of Petroleum Engineers Journal, 19:333-343, 1979.

[24] Y. Xia, M. Plummer, R. Podgorney, and A. Ghassemi. An Assessment of Some Design Constraints on Heat Production of a 3D Conceptual EGS Model Using an Open-Source Geothermal Reservoir Simulation Code. In PROCEEDINGS in the Forty-First Workshop on Geothermal Reservoir Engineering, pages 22-24. Stanford University, Stanford, California, 2016.

[25] J.W. Peterson, B.T. Murray, and G.F. Carey. Multi-resolution simulation of double-diffusive convection in porous media. International Journal of Numerical Methods for Heat \& Fluid Flow, 20(1):37-65, 2010.

[26] A. Masud and T. J. R. Hughes. A stabilized mixed finite element method for Darcy flow. Comput. Methods Appl. Mech. Engrg., 191:4341-4370, 2002. 


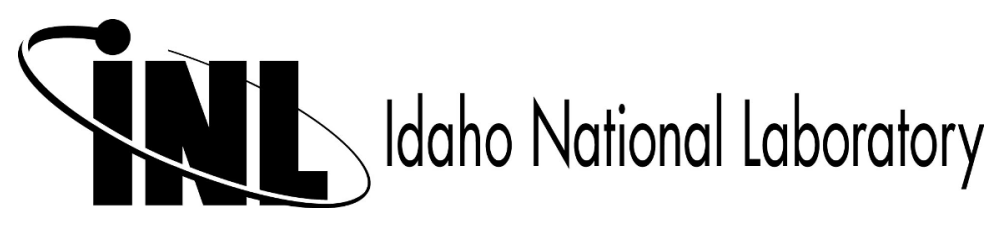

